\title{
Journal of Stochastic Analysis
}

Volume 2 | Number 1

Article 1

March 2021

\section{Fuzzy p-Value of Hypotheses Tests with Crisp Data Using Non- Asymptotic Fuzzy Estimators}

Nikos Mylonas

Democritus University of Thrace, Kimeria, Xanthi, 67100, Greece, nimylona@civil.duth.gr

Basil K. Papadopoulos

Democritus University of Thrace, Vas. Sofias 12, 67100 Xanthi, GREECE, papadob@civil.duth.gr

Follow this and additional works at: https://digitalcommons.Isu.edu/josa

Part of the Analysis Commons, and the Other Mathematics Commons

\section{Recommended Citation}

Mylonas, Nikos and Papadopoulos, Basil K. (2021) "Fuzzy p-Value of Hypotheses Tests with Crisp Data Using Non-Asymptotic Fuzzy Estimators," Journal of Stochastic Analysis: Vol. 2 : No. 1 , Article 1.

DOI: 10.31390/josa.2.1.01

Available at: https://digitalcommons.Isu.edu/josa/vol2/iss1/1 


\title{
FUZZY P-VALUE OF HYPOTHESES TESTS WITH CRISP DATA USING NON-ASYMPTOTIC FUZZY ESTIMATORS
}

\author{
NIKOS MYLONAS AND BASIL K. PAPADOPOULOS*
}

\begin{abstract}
In a fuzzy hypothesis test the null hypothesis is rejected or accepted with a degree of rejection or acceptance. A way to carry out such a test is to use fuzzy significance level and fuzzy $p$-value, which is a fuzzy number constructed by a fuzzy estimator. This approach is particularly useful in critical situations, where subtle comparisons between almost equal statistical quantities have to be made. In such cases the fuzzy hypotheses tests give better results than the crisp tests, since they give us the possibility of partial rejection or acceptance of $H_{0}$ using a degree of rejection or acceptance obtained by ordering the fuzzy numbers of p-value and significance level.
\end{abstract}

\section{Introduction}

In classical crisp statistics the problem of testing a hypothesis for a parameter $\theta$ of the distribution of a random variable $X$ is to decide whether to reject or accept at a significance level $\gamma$ the null hypothesis $H_{0}: \theta=\theta_{0}$ against the alternative hypothesis $H_{1}$ from a random sample of observations of $X$ using a test statistic $U$ which is evaluated for the sample observations, resulting in a value $u$. The space of possible values of $U$ is decomposed into a rejection region and its complement, the acceptance region. Depending on the alternative hypothesis $H_{1}$, the rejection region has one of the following forms [6]:

a) $U \leq u_{c 1}$, if the alternative hypothesis is $H_{1}: \theta<\theta_{0}$ (one sided test from the left), where

$$
P\left(U \leq u_{c 1}\right)=\gamma
$$

b) $U \geq u_{c 2}$, if the alternative hypothesis is $H_{1}: \theta>\theta_{0}$ (one sided test from the right), where

$$
P\left(U \geq u_{c 2}\right)=\gamma
$$

c) $U \leq u_{c 3}$ or $U \geq u_{c 4}$, if the alternative hypothesis is $H_{1}: \theta \neq \theta_{0}$ (two sided test), where

$$
P\left(U \leq u_{c 3}\right)=\frac{\gamma}{2} \quad \text { and } \quad P\left(U \geq u_{c 4}\right)=\frac{\gamma}{2}
$$

where $u_{c i}$ the critical values of the test, which are determined by the distribution of $U$. So, $H_{0}$ is rejected, if the value $u$ of the test statistic is in the rejection region and is not rejected, if $u$ is in the acceptance region.

Received 2020-10-23; Accepted 2020-11-27; Communicated by A. Boukas. 2010 Mathematics Subject Classification. Primary 62-XX; Secondary 62A86.

Key words and phrases. Fuzzy p-value, degree of rejection or acceptance, fuzzy hypotheses tests, non-asymptotic fuzzy estimators.

* Corresponding author. 
An equivalent way to test the null hypothesis $H_{0}$ against the alternative hypothesis $H_{1}$ is to calculate the p-value, which is defined for the above cases (a), (b), and (c) as ( $u$ is the sample value of the test statistic) [6]:

$$
\text { a) } p=P(U \leq u), \quad \text { b) } p=P(U \geq u) \text {, c) } p=2 \min \{P(U \leq u), P(U \geq u)\}
$$

If the p-value is less or equal to $\gamma$, then $H_{0}$ is rejected at the significance level $\gamma$, otherwise $H_{0}$ is not rejected.

Many approaches have been developed for fuzzy hypotheses testing. Among them [13] and [5] deal with the problem of hypotheses testing when the hypotheses are fuzzy from crisp or fuzzy data respectively using the concept of fuzzy $p$-value.

In [2] a method for testing crisp hypotheses has been proposed, which uses fuzzy test statistics obtained by fuzzy estimators produced by a set of confidence intervals and fuzzy critical values. In [12], from the various types of existing fuzzy estimators we use the well known non-asymptotic fuzzy estimators of [14] for the construction of the fuzzy test statistics. These are more convenient, because they are functions, whereas the fuzzy estimators of [2] are not functions, since they contain two small vertical segments.

Continuing this work, we present in this article a method for testing crisp hypotheses using fuzzy $p$-value $\bar{P}$ obtained by fuzzy test statistics, which are constructed by non-asymptotic fuzzy estimators. Fuzzifying the significance level $\gamma$ to $\bar{S}$ and using fuzzy ordering between $\bar{P}$ and $\bar{S}$, we decide for the rejection or acceptance of $H_{0}$ at the significance level $\gamma$ with a degree of confidence $d$, as explained in Sections 1.1 and 2.

If the $p$-value of the test is close to the significance level, then the crisp hypothesis test is unstable, since two similar samples may lead to contradicted result (one gives rejection and the other acceptance of $H_{0}$ ), as in Examples 3 and 6 . This problem does not exist in the respective fuzzy test in which we use a fuzzy test statistic $\bar{U}$ produced by a fuzzy estimator $\bar{\theta}$ of $\theta$ (instead of a crisp estimator) and a degree of rejection or acceptance of $H_{0}$ [12]. In such case a fuzzy hypothesis test gives similar results for the two similar samples: very low degree of rejection or acceptance, which means "no decision".

1.1. Ordering fuzzy numbers. The fuzzy hypotheses tests are based on ordering fuzzy numbers, for which we will use one of the several procedures used ([2],[3],[8],[9],[10]), according to which the degree of the inequality $A \leq B$ that counts the degree to which the fuzzy number $A$ is less or equal than the fuzzy number $B$ is defined as [2]

$$
v(A \leq B)=\max \{\min (A(x), B(y)) \quad x \leq y\}
$$

If $v(A \leq B)=1$, then the degree of confidence (truth-value) of $A<B$ is defined as

$$
v(A<B)=d \Leftrightarrow v(A \leq B)=1 \quad \text { and } \quad v(B \leq A)=1-d
$$

If $v(B \leq A)=1$, then the truth-value of $B<A$ is defined as

$$
v(B<A)=d \Leftrightarrow v(B \leq A)=1 \text { and } v(A \leq B)=1-d
$$

The truth-value $\eta$ of $A \approx B$ is defined as

$$
v(A \approx B)=\eta \Leftrightarrow v(A \leq B)=1 \text { and } v(B \leq A)=\eta
$$




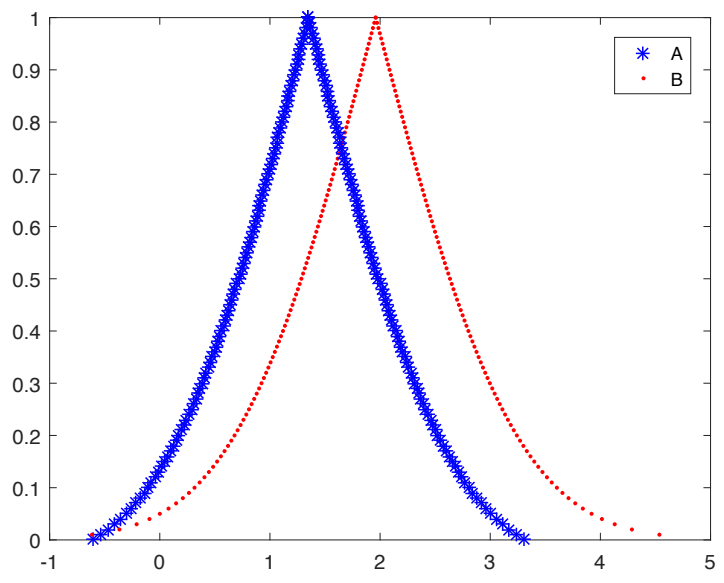

FiguRE 1. Ordering triangular shaped fuzzy numbers

$$
\text { or } v(B \leq A)=1 \text { and } v(A \leq B)=\eta
$$

In the case of the triangular shaped fuzzy numbers of Figure 1, which appears often in fuzzy hypotheses tests, we can see that:

$v(A \leq B)=1$ according to (1.2), since the core of $A$ is at the left of the core of $B$, $v(B \leq A)=y_{0}$ according to (1.2), where $y_{0}$ the truth level of the point of intersection of the right part of $A$ and the left part of $B$.

Therefore according to (1.3) and (1.5),

$$
v(A<B)=1-y_{0} \quad \text { and } \quad v(A \approx B)=y_{0}
$$

\section{Fuzzy p-value, Fuzzy Significance Level and Degree of Rejection or Acceptance of $H_{0}$}

Using Zadeh's extension principle [15], a fuzzy p-value is defined as a fuzzy number which is found by its $\alpha$-cuts.

In a fuzzy test of the null hypothesis $H_{0}: \theta=\theta_{0}$ for the parameter $\theta$ of a distribution from a random sample of observations we use a fuzzy test statistic $\bar{U}$ produced by a fuzzy estimator $\bar{\theta}$ of $\theta$. So, the $\alpha$-cuts of the fuzzy $p$-value of the test as described in [5] are:

a) for one sided test from the left (alternative hypothesis $H_{1}: \theta<\theta_{0}$ ):

$$
\bar{P}[\alpha]=\left[\operatorname{Pr}\left(U \leq \bar{U}_{l}[\alpha]\right), \operatorname{Pr}\left(U \leq \bar{U}_{r}[\alpha]\right)\right]
$$

where $U$ the test statistic and $\bar{U}_{l}[\alpha]$ and $\bar{U}_{r}[\alpha]$ the $\alpha$-cuts of the fuzzy test statistic $\bar{U}$ for the given sample,

b) for one sided test from the right (alternative hypothesis $H_{1}: \theta>\theta_{0}$ ):

$$
\bar{P}[\alpha]=\left[\operatorname{Pr}\left(U \geq \bar{U}_{r}[\alpha]\right), \operatorname{Pr}\left(U \geq \bar{U}_{l}[\alpha]\right)\right]
$$


c) for two sided test (alternative hypothesis $H_{1}: \theta \neq \theta_{0}$ ):

$$
\bar{P}[\alpha]=\left\{\begin{aligned}
{\left[2\left(\operatorname{Pr}\left(U \leq \bar{U}_{l}[\alpha]\right)\right),\right.} & \\
\left.\min \left\{1,2\left(\operatorname{Pr}\left(U \leq \bar{U}_{r}[\alpha]\right)\right)\right\}\right], & \\
{\left[2 \operatorname{Pr}\left(U \geq \bar{U}_{r}[\alpha]\right)\right), } & \\
\left.\min \left\{1,2 \operatorname{Pr}\left(U \geq \bar{U}_{l}[\alpha]\right)\right\}\right], & u_{0} \geq m_{U}
\end{aligned}\right.
$$

where $m_{U}$ the median of the distribution of $U$ under the null hypothesis $H_{0}$ and $u_{0}$ the core of the fuzzy test statistic $\bar{U}$ (where $\bar{U}\left(u_{0}\right)=1$ ).

Since the p-value is fuzzy, the significance level has to be a fuzzy set $\bar{S}$, too.

A simple way to fuzzify a significance level $\gamma$ is to use a triangular fuzzy numbers of one of the forms [7]

$$
\text { (a) } \bar{S}=T(0, \gamma, 2 \gamma) \quad \text { or } \quad(b) \bar{S}=T(\gamma-c, \gamma, 2 \gamma+c), c \text { constant }
$$

which expresses the notion "the significance level is approximately $\gamma$ ".

So, using fuzzy ordering between the fuzzy numbers $\bar{P}$ and $\bar{S}$ (Section 1.1):

$H_{0}$ is rejected at the significance level $\gamma$ with degree of rejection $d$, if the truth value of $\bar{P}<\bar{S}$ is $d$,

$H_{0}$ is accepted (not rejected) at the significance level $\gamma$ with degree of acceptance $d$ if the truth value of $\bar{P}>\bar{S}$ is $d$,

if the truth value of $\bar{P} \approx \bar{S}$ is $d_{1}$, then no decision can be made on the rejection or the acceptance of $H_{0}$ at the significance level $\gamma$ with any degree of confidence $d>1-d_{1}$.

\section{Fuzzy p-value of the Mean of a Normal Distribution or of Any Distribution From Large Sample}

In the following subsections we present the development of the fuzzy $p$-value of hypotheses tests for the mean of a normal random variable with known variance, of a random variable that follows any distribution from a large sample and for the equality of the means of two normal random variables with known variances, using fuzzy statistics constructed by the non-asymptotic fuzzy estimator of the mean developed in [2] and [14].

3.1. Fuzzy p-value of the hypotheses tests for the mean of a normal random variable with known variance. We test at significance level $\gamma$ the null hypothesis that the mean $\mu$ of a random variable $X$ that follows normal distribution with known variance $\sigma^{2}$ is equal to $\mu_{0}$,

$$
H_{0}: \mu=\mu_{0}
$$

using a random sample of observations of $X$ of size $n$ with mean $\bar{x}$.

3.1.1. Two sided test. In the two sided test of $H_{0}$ the alternative is

$$
H_{1}: \mu \neq \mu_{0}
$$

In the crisp case we use the statistic [6]

$$
Z=\frac{\bar{X}-\mu_{0}}{\sigma / \sqrt{n}}
$$


where $\bar{X}$ is the statistic of the sample mean. It is known [6] that under the null hypothesis $\left(\mu=\mu_{0}\right) Z$ follows the standard normal distribution $N(0,1)$, so from a random sample of observations $H_{0}$ is rejected if $p \leq \gamma$, where according to (1.1)

$$
p= \begin{cases}2 \Phi\left(z_{0}\right), & z_{0} \leq 0 \\ 2\left(1-\Phi\left(z_{0}\right)\right), & z_{0}>0\end{cases}
$$

the crisp $p$-value of the test, $\Phi(z)$ the distribution function of the standard normal distribution and

$$
z_{0}=\frac{\bar{x}-\mu_{0}}{\sigma / \sqrt{n}}
$$

the sample value of the statistic (3.1). If $p>\gamma$, then $H_{0}$ is not rejected.

In the fuzzy case, the test of $H_{0}$ is based on the fuzzy statistic

$$
\bar{Z}=\frac{\bar{\mu}-\mu_{0}}{\sigma / \sqrt{n}}
$$

which is generated by substituting $\bar{X}$ in (3.1) with a fuzzy estimator $\bar{\mu}$ of the mean [2].

In our approach we use the non-asymptotic fuzzy estimator $\bar{\mu}$ the $\alpha$-cuts of which for the given sample are [14]

$$
\bar{\mu}[\alpha]=\left[\bar{x}-z_{h(\alpha)} \frac{\sigma}{\sqrt{n}}, \bar{x}+z_{h(\alpha)} \frac{\sigma}{\sqrt{n}}\right], \quad \alpha \in[0,1]
$$

where $\bar{x}$ the sample mean,

$$
h:[0,1] \rightarrow\left[\frac{\gamma_{0}}{2}, \frac{1}{2}\right] \quad h(\alpha)=\left(\frac{1}{2}-\frac{\gamma_{0}}{2}\right) \alpha+\frac{\gamma_{0}}{2}, \quad \gamma_{0}=0.01
$$

and

$$
z_{h(\alpha)}=\Phi^{-1}(1-h(\alpha))
$$

where $\Phi^{-1}$ the inverse distribution function of the standard normal distribution. From (3.1)-(3.6) and interval arithmetics follows that the $\alpha$-cuts of the fuzzy statistic $\bar{Z}$ for the given sample are [2]

$$
\bar{Z}_{0}[\alpha]=\left[z_{0}-z_{h(\alpha)}, z_{0}+z_{h(\alpha)}\right], \quad \alpha \in[0,1]
$$

The median of the standard normal distribution is equal to its mean, which is zero, and the core of $\bar{Z}$ is obtained for $\alpha=1$ to be $z_{0}-z_{h(1)}=z_{0}+z_{h(1)}=z_{0}$, since according to $(3.5) h(1)=0.5$, so $z_{h(1)}=\Phi^{-1}(1-0.5)=0$. Hence, since the statistic $Z$ follows the standard normal distribution, according to (2.3) the $\alpha$-cuts of the $p$-value of this test are:

$$
\begin{aligned}
& \bar{P}[\alpha]=\left\{\begin{aligned}
{\left[2 \operatorname{Pr}\left(Z \leq z_{0}-z_{h(\alpha)}\right),\right.} & \\
\left.\min \left\{1,2 \operatorname{Pr}\left(Z \leq z_{0}+z_{h(\alpha)}\right)\right\}\right], & z_{0} \leq 0 \\
{\left[2\left(\operatorname{Pr}\left(Z \geq z_{0}+z_{h(\alpha)}\right)\right),\right.} & \\
\left.\min \left\{1,2 \operatorname{Pr}\left(Z \geq z_{0}-z_{h(\alpha)}\right)\right\}\right], & z_{0}>0
\end{aligned}\right. \\
& =\left\{\begin{array}{cc}
{\left[2 \Phi\left(z_{0}-z_{h(\alpha)}\right),\right.} & \\
\left.\min \left\{1,2 \Phi\left(z_{0}+z_{h(\alpha)}\right)\right\}\right], & \\
{\left[2\left(1-\Phi\left(z_{0}+z_{h(\alpha)}\right)\right),\right.} & \\
\left.\min \left\{1,2\left(1-\Phi\left(z_{0}-z_{h(\alpha)}\right)\right)\right\}\right], & z_{0}>0
\end{array}\right.
\end{aligned}
$$




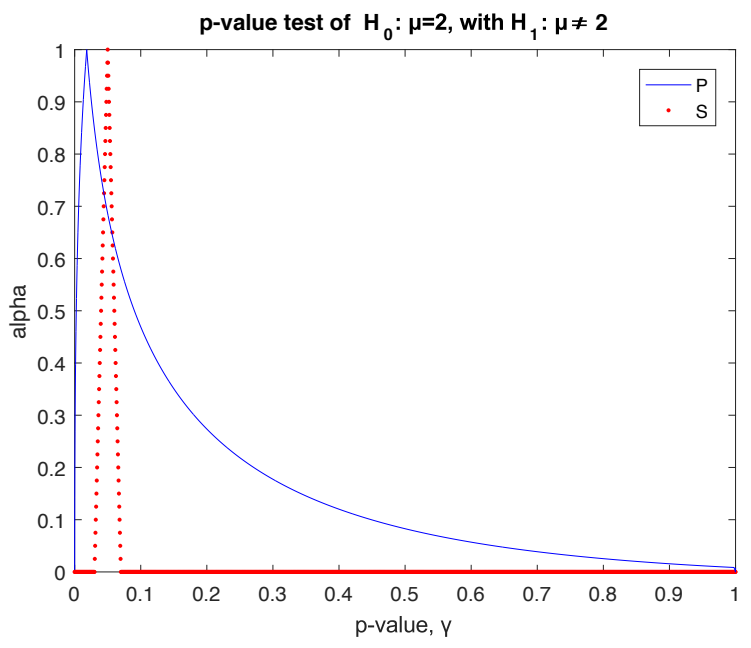

Figure 2. Fuzzy p-value $\bar{P}$ and significance level $\bar{S}$ for the two sided test of $H_{0}: \mu=2$ of Example 1

where $\Phi(z)$ the distribution function of the standard normal distribution and $z_{0}$ and $z_{h(\alpha)}$ are given by (3.3) and (3.6).

Using the fuzzy significance level $\bar{S}$ given in (2.4), $H_{0}$ is rejected at significance level $\gamma$ if $\bar{P}<\bar{S}$ and not rejected if $\bar{P}>\bar{S}$ with a degree of rejection or acceptance as described in Section 2.

3.1.2. One sided test. If the alternative is (one sided test from the left)

$$
H_{1}: \mu<\mu_{0},
$$

then according to (1.1) the crisp $p$-value is

$$
p=\Phi\left(z_{0}\right)
$$

According to (2.1) the $\alpha$-cuts of the $p$-value of this test are

$$
\begin{aligned}
\bar{P}[\alpha] & =\left[\operatorname{Pr}\left(Z \leq z_{0}-z_{h(\alpha)}\right), \operatorname{Pr}\left(Z \leq z_{0}+z_{h(\alpha)}\right)\right] \\
& =\left[\Phi\left(z_{0}-z_{h(\alpha)}\right), \Phi\left(z_{0}+z_{h(\alpha)}\right)\right]
\end{aligned}
$$

If the alternative is (one sided test from the right)

$$
H_{1}: \mu>\mu_{0},
$$

then according to (1.1) the crisp $p$-value is

$$
p=1-\Phi\left(z_{0}\right)
$$

According to (2.2) the $\alpha$-cuts of the $p$-value of this test are

$$
\begin{aligned}
\bar{P}[\alpha] & =\left[\operatorname{Pr}\left(Z \geq z_{0}+z_{h(\alpha)}\right), \operatorname{Pr}\left(Z \geq z_{0}-z_{h(\alpha)}\right)\right] \\
& =\left[1-\Phi\left(z_{0}+z_{h(\alpha)}\right), \quad 1-\Phi\left(z_{0}-z_{h(\alpha)}\right)\right]
\end{aligned}
$$




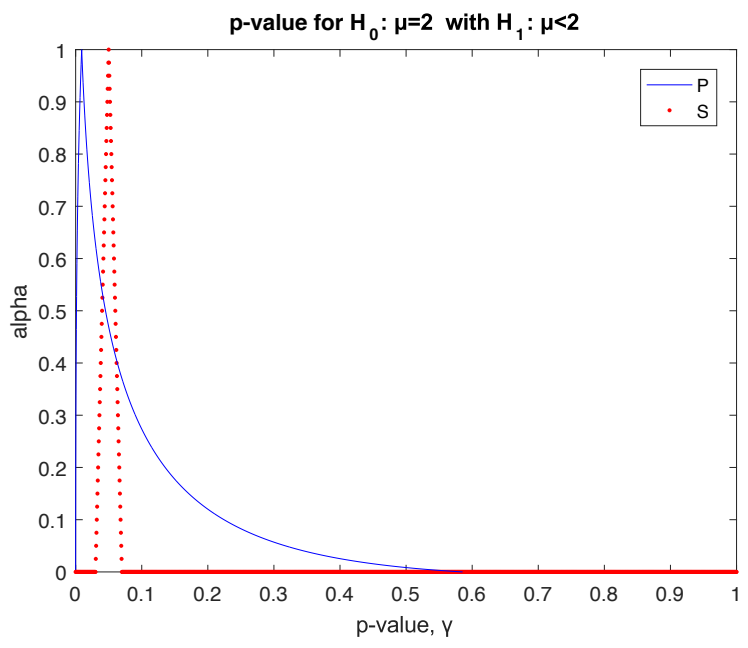

Figure 3 . Fuzzy $p$-value $\bar{P}$ and significance level $\bar{S}$ for the one sided test of $H_{0}: \mu=2$ with $H_{1}: \mu<2$ of Example 1

Example 1. a) We test the null hypothesis $H_{0}: \mu=2$ at significance level $\gamma=0.05$ with alternative $H_{1}: \mu \neq 2$ (two sided test) for the mean $\mu$ of a random variable $X$ that follows normal distribution with variance $\sigma^{2}=4$ using a random sample of $n=25$ observations with sample mean $\bar{x}=1,056$.

b) We test $H_{0}$ at significance level $\gamma=0.05$ with alternative $\left(H_{1}: \mu<2\right.$ one sided test from left) using the same sample.

a) In the crisp test the sample value of the statistic (3.1) is given by (3.3),

$$
z_{0}=\frac{1,056-2}{2 / \sqrt{25}}=-2.36
$$

so the crisp p-value of the two sided test is given by $(3.2)$,

$$
p=2 \Phi(-2.36)=0.018<\gamma=0.05
$$

Therefore $H_{0}$ is rejected by this crisp test.

For $\gamma=0.05,(3.5)$ and (3.6) give

$$
z_{h(\alpha)}=\Phi^{-1}\left(1-\left[\left(\frac{1}{2}-\frac{0.05}{2}\right) \alpha+\frac{0.05}{2}\right]\right)=0.98-0.48 a
$$

so according to (3.7), the $\alpha$-cuts of the fuzzy p-value are $\left(z_{0}=-2.36\right)$

$$
\begin{aligned}
\bar{P}[\alpha] & =[2 \Phi(-2.36-(0.98-0.48 a)), \min \{1,2 \Phi(-2.36+(0.98-0.48 a)\}] \\
& =[2(\Phi(-3.34+0.48 a)), \min \{1,2 \Phi(-1.38-0.48 a)\}] .
\end{aligned}
$$

According to (2.4b) the significance level $\gamma=0.05$ can be fuzzified to a triangular fuzzy number as

$$
\bar{S}(a)=T(\gamma-0.02, \gamma, \gamma+0.02)=T(0.03,0.05,0.07)
$$




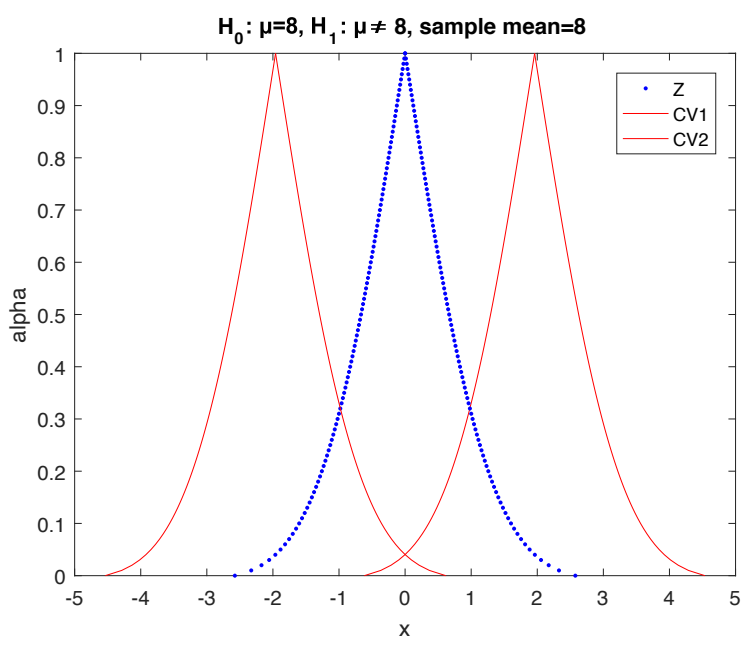

Figure 4 . Fuzzy statistic $\bar{Z}$ and critical values $\overline{C V}_{i}$ for the two sided test of Example 2

So implementing (3.10) and (3.11), we get the results of Figure 2, where the core of the fuzzy number $\bar{P}$ is at the left of the core of $\bar{S}$ and their point of intersection has $y_{0}=0.72$, so the truth value of $\bar{P}<\bar{S}$ is $1-0.72=0.28$. Therefore, $H_{0}$ is rejected the two sided test with degree of rejection 0.28 .

b) The crisp $p$-value of the one sided test from the left is

$$
p=\Phi(-2.36)=0.009<\gamma=0.05,
$$

so $H_{0}$ is rejected by this test.

Implementing (3.8) and (3.11), we get the results of Figure 3, where the core of the fuzzy number $\bar{P}$ is at the left of $\bar{S}$ and their point of intersection has $y_{0}=0.51$, so the truth value of $\bar{P}<\bar{S}$ is $1-0.51=0.49$. Therefore, $H_{0}$ is rejected by the one sided test from the left with degree of rejection 0.49 .

Example 2. We test at significance level 0.05 the null hypothesis $H_{0}: \mu=8$ with alternative $H_{1}: \mu \neq 8$ (two sided test) for the mean $\mu$ of a random variable $X$ that follows normal distribution with variance $\sigma^{2}=4$ from a sample of $n=25$ observations with sample mean $\bar{x}=8$ with two sided fuzzy test which uses:

a) fuzzy critical values, developed in [2] and [12],

b) fuzzy $p$-value.

The value of the test statistic $Z$ is

$$
z_{0}=\frac{8-8}{2 / \sqrt{25}}=0
$$

Since

$$
z_{0}=0<z_{0.05 / 2}=1.96
$$




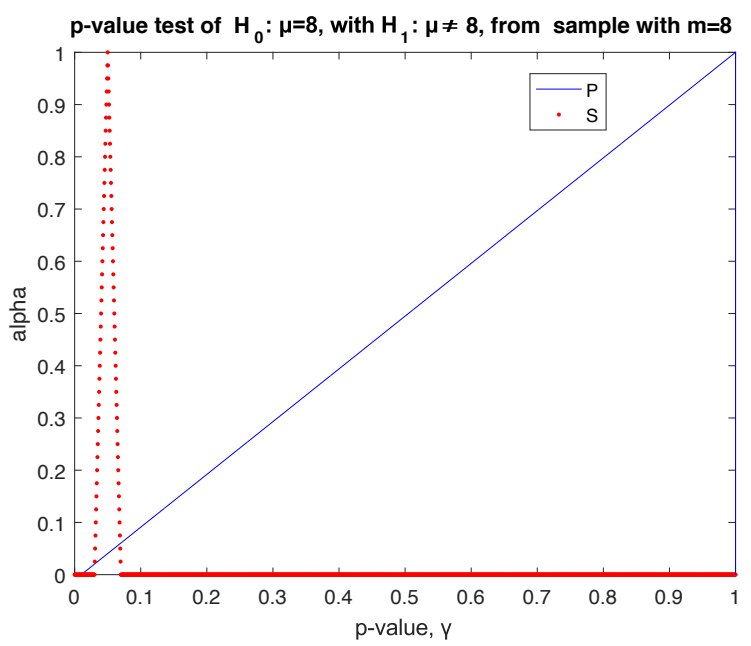

Figure 5. Fuzzy $p$-value $\bar{P}$ and significance level $\bar{S}$ for the two sided test of Example 2

$H_{0}$ is not rejected by this crisp test.

Also, the crisp $p$-value of the test is given by (3.2),

$$
p=2 \Phi(0)=2 \cdot 0.5=1>\gamma=0.05
$$

In this case the crisp test gives no rejection of $H_{0}$ with the largest possible difference between the test statistic and the critical values since the value of the test statistic is exactly in the middle of the no rejection region (the crisp $p$-value has its maximum possible value, one), so it is the best case for acceptance of $H_{0}$.

a) Applying the two sided fuzzy test of [12], which uses the fuzzy critical values of [2], we obtain the results of Figure 4, where the point of intersection of the fuzzy numbers $\bar{Z}$ and $\overline{C V}_{1}$ is $y_{1}=0.31$ and of $\bar{Z}$ and $\overline{C V}_{2}$ is $y_{2}=0.32$, so

$$
\min \left(v\left(\bar{Z}>\overline{C V}_{1}\right), v\left(\bar{Z}<\overline{C V}_{2}\right)\right)=\min \left(1-y_{1}, 1-y_{2}\right)=0.68
$$

Therefore, $H_{0}$ is accepted by this test with degree of acceptance $d=0.68$.

b) Implementing (3.7) and (3.11) for the two sided test of $H_{0}$, we get the results of Figure 5, where the core of the fuzzy number $\bar{P}$ is at the right of $\bar{S}$ and their point of intersection has $y_{0}=0.05$, so the truth value of $\bar{P}>\bar{S}$ is $1-0.05=0.95$. Therefore, $H_{0}$ is accepted by this test with degree of acceptance 0.95 .

\subsection{Fuzzy p-value of hypotheses tests for the mean of a random variable} from large sample. We test at significance level $\gamma$ the null hypothesis that the mean $\mu$ of a random variable $X$ is equal to $\mu_{0}$

$$
H_{0}: \mu=\mu_{0}
$$

using a random sample of observations of $X$ of large size $n(n>30)$. 


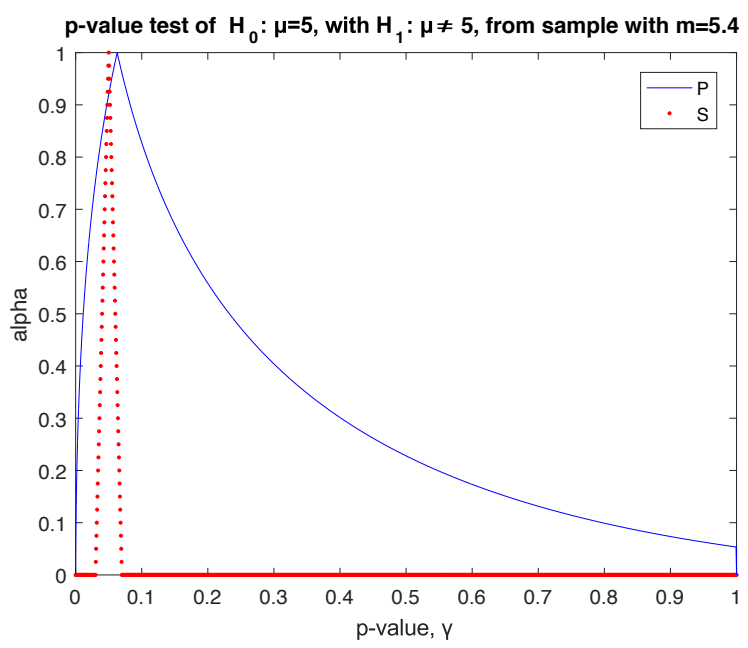

Figure 6 . Fuzzy p-value $\bar{P}$ and significance level $\bar{S}$ for the two sided test of $H_{0}: \mu=5$ of Example 3 from a sample with $\bar{x}=5.4$

In the crisp case we test $H_{0}$ using the statistic

$$
Z=\frac{\bar{X}-\mu_{0}}{s / \sqrt{n}}
$$

where $\bar{X}$ the statistic of the sample mean and $s$ the sample standard deviation. It is known [6] that under the null hypothesis $\left(\mu=\mu_{0}\right) Z$ follows the standard normal distribution $N(0,1)$ according to the central limit theorem, so $H_{0}$ is rejected if $p \leq \gamma$, where the crisp $p$-value of the test is found as in Section 3.1 for the sample value of the statistic (3.12),

$$
z_{0}=\frac{\bar{x}-\mu_{0}}{s / \sqrt{n}}
$$

If $p>\gamma$, then $H_{0}$ is not rejected.

In the fuzzy case, for the test of $H_{0}$ we use the fuzzy statistic

$$
\bar{Z}=\frac{\bar{\mu}-\mu_{0}}{s / \sqrt{n}}
$$

which is generated by substituting $\bar{X}$ in (3.12) with the non-asymptotic fuzzy estimator of the mean of [14], the $\alpha$-cuts of which for the given sample are

$$
\bar{\mu}[\alpha]=\left[\bar{x}-z_{h(\alpha)} \frac{s}{\sqrt{n}}, \bar{x}+z_{h(\alpha)} \frac{s}{\sqrt{n}}\right], \quad \alpha \in[0,1]
$$

So, the $\alpha$-cuts of the fuzzy $p$-value are given by (3.7) for the two sided test and by (3.8) or (3.9) for the one sided tests, for the value $z_{0}$ of (3.13).

Example 3. We test the null hypothesis $H_{0}: \mu=5$ at significance level $\gamma=0.05$ with alternative the $H_{1}: \mu \neq 5$ (two sided test) for the mean $\mu$ of a random variable $X$ for two random large samples of $n=100$ observations with sample 


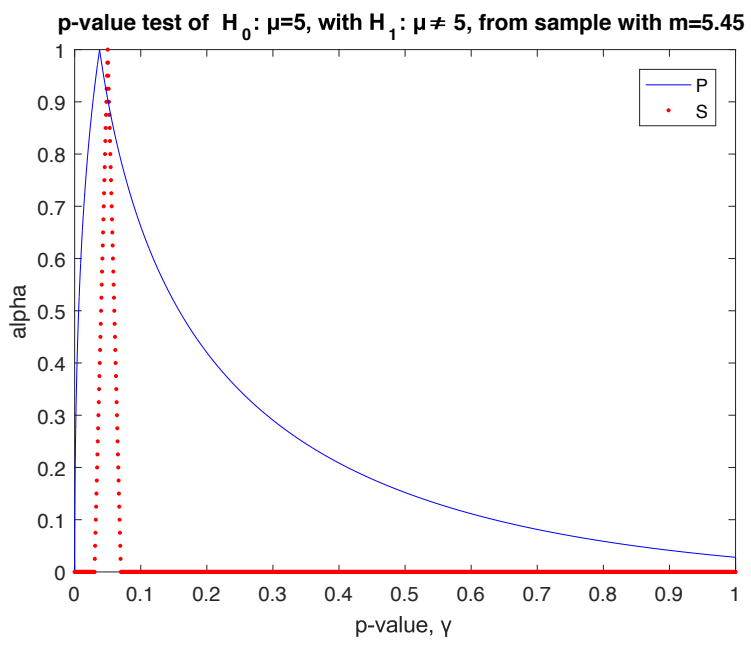

Figure 7. Fuzzy p-value $\bar{P}$ and significance level $\bar{S}$ for the two sided test of $H_{0}: \mu=5.45$ of Example 3 from a sample with $\bar{x}=5.45$

means and variances:

a) $\bar{x}=5.40$ and $s^{2}=4.62, \quad$ b) $\bar{x}_{2}=5.45$ and $s^{2}=4.69$

We evaluate the value of the statistic (3.12) for the first sample from (3.13)

$$
z_{0}=\frac{5.4-5}{\sqrt{4.62} / \sqrt{100}}=1.86
$$

so the crisp $p$-value of the test is found by (3.2) to be

$$
p=2(1-\Phi(1.86))=0.063>\gamma=0.05
$$

Therefore $H_{0}$ is not rejected by this test.

For the second sample, which has sample mean and variance $\bar{x}_{2}=5.45$ and $s^{2}=4.69$ (very close to these of the first sample), (3.13) gives

$$
z_{0}=\frac{5.45-5}{\sqrt{4.69} / \sqrt{100}}=2.08
$$

so the crisp $p$-value of the test is found by (3.2) to be

$$
p=2(1-\Phi(2.08))=0.038<\gamma=0.05
$$

Therefore $H_{0}$ is rejected by this test.

a) Implementing (3.7) for the first sample fuzzifying the significance level to a triangular fuzzy number as in (3.11), we get the results of Figure 6 , where the point of intersection of the fuzzy numbers $\bar{P}$ and $\bar{S}$ is $y_{0}=0.93$. This according to $(1.5)$ means that $v(\bar{P} \approx \bar{S})=0.93$, so according to Section 2 , we cannot make a decision on rejecting or not $H_{0}$ by this test with any degree of confidence $d>0.07$.

b) For the second sample $(\bar{x}=5.45)$, we plot the fuzzy numbers $\bar{P}$ and $\bar{S}$ in Figure 7 , where their point of intersection has $y_{0}=0.94$, so $v(\bar{P} \approx \bar{S})=0.91$. 


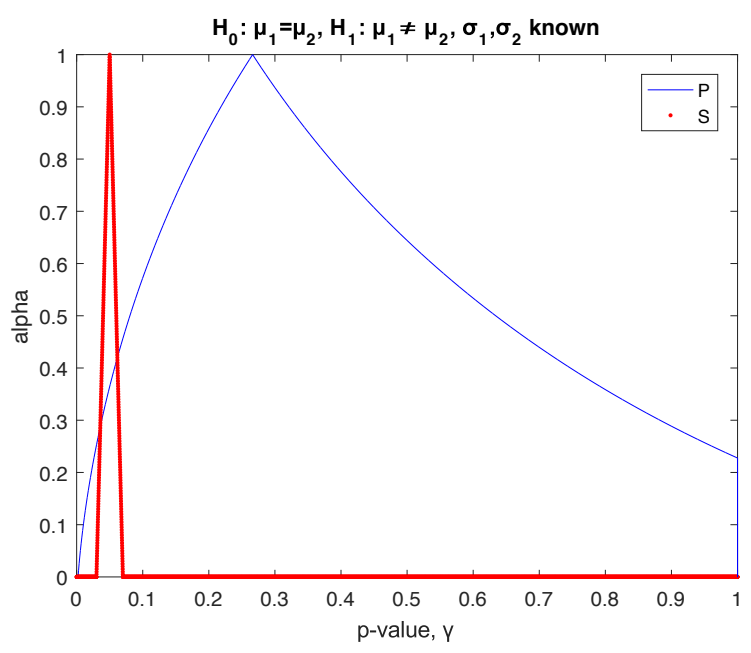

Figure 8. Fuzzy p-value $\bar{P}$ and significance level $\bar{S}$ of the two sided test of $H_{0}: \mu_{1}=\mu_{2}$ of Example 4

Therefore, we cannot make a decision on rejecting or not $H_{0}$ from this test with any degree of confidence $d>0.09$.

3.3. Fuzzy p-value of the tests of $H_{0}: \mu_{1}=\mu_{2}$, for the means $\mu_{1}, \mu_{2}$ of two normal random variables with known variances. We test at significance level $\gamma$ the null hypothesis that the means $\mu_{1}$ and $\mu_{2}$ of two random variables $X_{1}$ and $X_{2}$ that follow normal distributions with variances $\sigma_{1}^{2}$ and $\sigma_{2}^{2}$ are equal,

$$
H_{0}: \mu_{1}=\mu_{2}
$$

using two independent random samples of observations of $X_{1}$ and $X_{2}$ of sizes $n_{1}$ and $n_{2}$ with sample means and variances $\bar{x}_{1}, \bar{x}_{2}$ and $s_{1}^{2}, s_{2}^{2}$.

It is known [6] that under the null hypothesis (equal means) the test statistic

$$
Z=\frac{\bar{X}_{1}-\bar{X}_{2}}{\sigma_{0}} \quad \text { where } \quad \sigma_{0}=\sqrt{\frac{s_{1}^{2}}{n_{1}}+\frac{s_{2}^{2}}{n_{2}}}
$$

$\left(\bar{X}_{1}, \bar{X}_{2}\right.$ the statistics of the sample means) follows standard normal distribution $N(0,1)$, so $H_{0}$ is rejected from a random sample of observations if $p \leq \gamma$, where the crisp $p$-value of the test is found as in Section 3.1 for the value of the statistic (3.15) for the given sample,

$$
z_{0}=\frac{\bar{x}_{1}-\bar{x}_{2}}{\sigma_{0}}
$$

If $p>\gamma$, then $H_{0}$ is not rejected.

In the fuzzy test of $H_{0}$, we use the fuzzy statistic

$$
\bar{Z}=\frac{\bar{\mu}_{12}}{\sigma_{0}}
$$


that is generated by substituting $\bar{X}_{1}-\bar{X}_{2}$ in (3.15) with the non-asymptotic fuzzy estimator $\bar{\mu}_{12}$ of the difference of the sample means [2].

The $\alpha$-cuts of $\bar{\mu}_{12}$ for the given sample are found as in Section 3.1 to be

$$
\bar{Z}[\alpha]=\left[z_{0}-z_{h(\alpha)}, z_{0}+z_{h(\alpha)}\right], \quad \alpha \in(0,1]
$$

So, the $\alpha$-cuts of the fuzzy p-value are given by (3.7) for the two sided test and by (3.8) or (3.9) for the one sided tests for the value $z_{0}$ of (3.16).

Example 4. We test the null hypothesis $H_{0}: \mu_{1}=\mu_{2}$ at significance level $\gamma=0.05$ with alternative the $H_{1}: \mu_{1} \neq \mu_{2}$ (two sided test) for the means $\mu_{1}$ and $\mu_{2}$ of the variables $X_{1}$ and $X_{2}$ that follow normal distributions with variances $\sigma_{1}^{2}=5.2$ and $\sigma_{2}^{2}=3.7$ from two independent random samples of observations of sizes $n_{1}=15$ and $n_{2}=8$ and sample means $\bar{x}_{1}=50.2$ and $\bar{x}_{2}=51.1$ with two sided fuzzy test which uses:

a) fuzzy $p$-value,

b) fuzzy critical values, developed in [2] and [12].

According to (3.16), the value of the test statistic (3.15) is

$$
z_{0}=\frac{50.2-51.1}{\sqrt{\frac{5.2}{15}+\frac{3.7}{8}}}=-1
$$

so the crisp p-value of the test is given by (3.2)

$$
p=2 \Phi(-1)=0.387>\gamma=0.05
$$

Therefore $H_{0}$ is not rejected by the crisp test.

a) Implementing (3.7) for $z_{0}=-1$ and fuzzifying the significance level to a triangular fuzzy number as in (3.11), we get the results of Figure 8, where the core of $\bar{P}$ is at the right of the core of $\bar{S}$ and their point of intersection has $y_{0}=0.43$, so $v(\bar{P}>\bar{S})=1-0.43=0.57$. Therefore $H_{0}$ is accepted by this test with degree of acceptance 0.57 .

b) Applying the fuzzy test which uses fuzzy critical values developed in [2] and [12], we get the results of Figure 9, where the core of $\bar{Z}_{0}$ is between the cores of $\overline{C V}_{1}$ and $\overline{C V}_{2}$ and the point of intersection of $\bar{Z}_{0}$ and $\overline{C V}_{1}$ has truth value $y_{1}=0.63$ and of $\bar{Z}_{0}$ and $\overline{C V} \bar{V}_{2}, y_{2}=0.12$, so that

$$
\min \left(v\left(\bar{Z}_{0}>\overline{C V}_{1}\right), v\left(\bar{Z}_{0}<\overline{C V}_{2}\right)\right)=\min \left(1-y_{1}, 1-y_{2}\right)=0.37
$$

Therefore, $H_{0}$ is accepted by this test with degree of acceptance $d=0.37$.

\section{4. p-value of the Test for the Variance of a Normal Random Variable}

In this section we present the hypotheses testing of the variance of a normal random variable and in the next section of the variances of two normal random variables using fuzzy $p$-value produced by fuzzy statistics [2] constructed by the non-asymptotic fuzzy estimator of the variance given in [14].

We test at significance level $\gamma$ the null hypothesis that the variance $\sigma^{2}$ of a normal random variable $X$ is equal to $\sigma_{0}^{2}$,

$$
H_{0}: \sigma^{2}=\sigma_{0}^{2}
$$




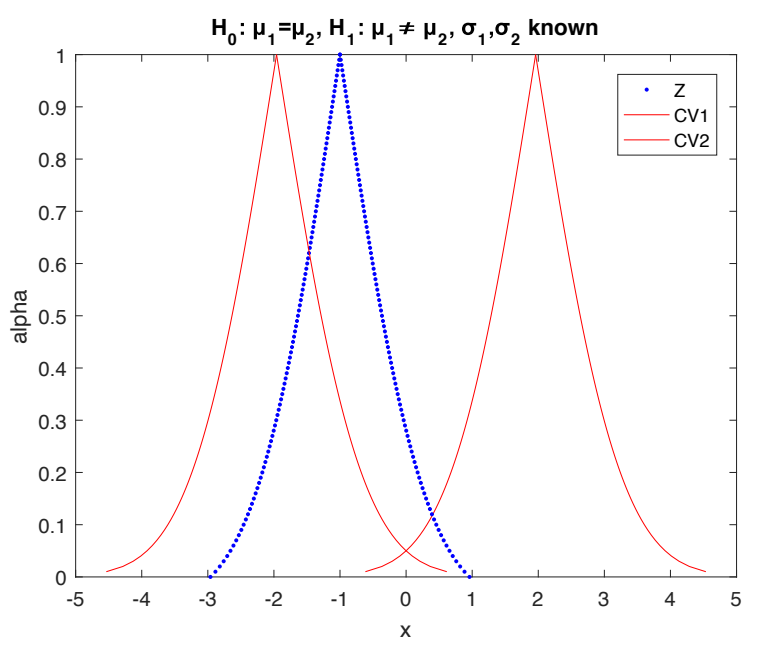

Figure 9. The fuzzy test statistic $Z$ and critical values for the test of Example 4

using a random sample of $n$ observations of $X$ with sample variance $s^{2}$. In the crisp case we test $H_{0}$ using the test statistic [6]

$$
\chi^{2}=\frac{(n-1) S^{2}}{\sigma_{0}^{2}}
$$

where $S^{2}$ is the statistic of the sample variance.

It is known that under the null hypothesis $\left(\sigma^{2}=\sigma_{0}\right)$ the statistic $\chi^{2}$ follows $\chi_{n-1}^{2}$ distribution with $n-1$ degrees of freedom [6], so from a sample of observations $H_{0}$ is rejected if $p \leq \gamma$, where the crisp $p$-value of the test according to (1.1) is: for the one sided test from the left,

$$
p=F_{n-1}\left(\chi_{0}^{2}\right)
$$

for the one sided test from the right,

$$
p=1-F_{n-1}\left(\chi_{0}^{2}\right)
$$

for the two sided test,

$$
p=\min \left\{2 F_{n-1}\left(\chi_{0}^{2}\right), 2\left(1-F_{n-1}\left(\chi_{0}^{2}\right)\right)\right\}
$$

where $F_{n-1}$ the distribution function of the $\chi_{n-1}^{2}$ distribution and

$$
\chi_{0}^{2}=\frac{(n-1) s^{2}}{\sigma_{0}^{2}}
$$

the value of the statistic $\chi^{2}$ for the given sample. If $p>\gamma$, then $H_{0}$ is not rejected.

The fuzzy statistic of this test is derived by substituting $S^{2}$ in $\chi^{2}$ with a fuzzy estimator $\overline{\sigma^{2}}$ of the variance,

$$
\overline{\chi^{2}}=\frac{(n-1) \overline{\sigma^{2}}}{\sigma_{0}^{2}}
$$




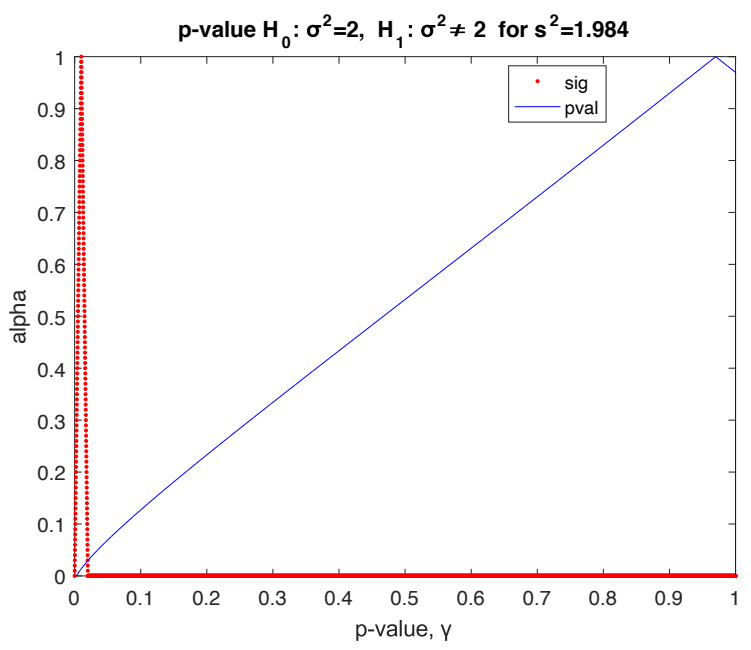

Figure 10. Fuzzy p-value $\bar{P}$ and significance level $\bar{S}$ of the two sided test of Example 5

In our approach we use the non-asymptotic fuzzy estimator, the $\alpha$-cuts of which for the given sample are found in [14] to be

$$
\overline{\sigma^{2}}[\alpha]=\left[\frac{(n-1) s^{2}}{\chi_{R, h(\alpha) ; n-1}^{2}}, \frac{(n-1) s^{2}}{\chi_{L, h(\alpha) ; n-1}^{2}}\right]
$$

where

$$
\chi_{L, h(\alpha) ; n-1}^{2}=F_{n-1}^{-1}(h(\alpha)) \quad \text { and } \quad \chi_{R, h(\alpha) ; n-1}^{2}=F_{n-1}^{-1}(1-h(\alpha))
$$

From (4.2)-(4.4) and interval arithmetics we deduce that the $\alpha$-cuts of $\overline{\chi^{2}}$ for the given sample are

$$
\overline{\chi^{2}}[\alpha]=\left[\frac{(n-1) \chi_{0}^{2}}{\chi_{R ; h(\alpha) ; n-1}^{2}}, \frac{(n-1) \chi_{0}^{2}}{\chi_{L ; h(\alpha) ; n-1}^{2}}\right]
$$

The core of $\overline{\chi^{2}}$ is obtained by (4.6) for $\alpha=1$ to be $(n-1) \chi_{0}^{2} / m$ (from (3.5) $h(1)=0.5$, so $\left.\chi_{R ; h(1) ; n-1}^{2}=\chi_{L ; h(1) ; n-1}^{2}=F_{n-1}^{-1}(0.5)=m\right)$, where $m$ the median of the $\chi_{n-1}^{2}$ distribution, so

$$
\frac{(n-1) \chi_{0}^{2}}{m}<m \Leftrightarrow \chi_{0}^{2}<\frac{m^{2}}{n-1}
$$




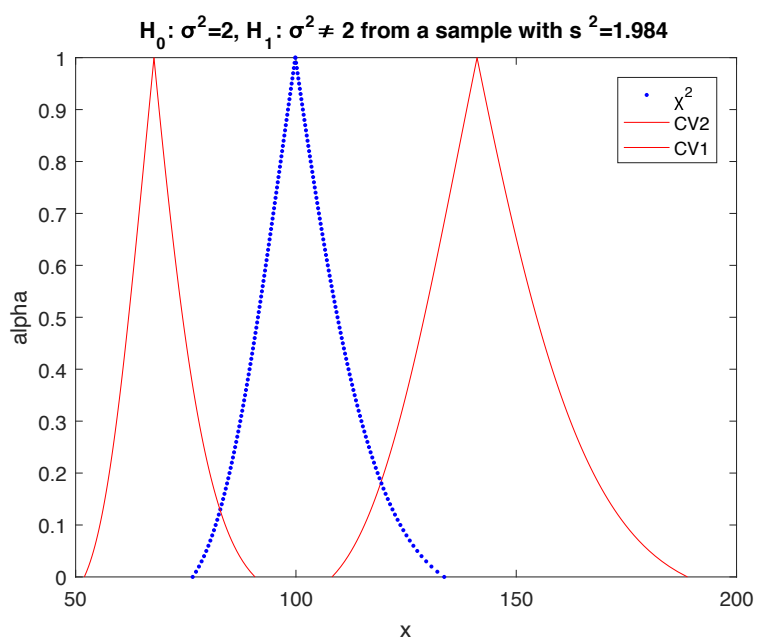

Figure 11 . Fuzzy statistic $\bar{Z}$ and critical values $\overline{C V}_{1}, \overline{C V}_{2}$ of the two sided test of Example 5

Therefore, according to (2.3) and (4.6) the $\alpha$-cuts of the fuzzy $p$-value of the two sided test of $H_{0}$ are

$$
\bar{P}[\alpha]=\left\{\begin{array}{l}
{\left[2 \operatorname{Pr}\left(\chi^{2}<\frac{(n-1) \chi_{0}^{2}}{\chi_{R ; h(\alpha) ; n-1}^{2}}\right),\right.} \\
\left.\min \left\{1,2 \operatorname{Pr}\left(\chi^{2}<\frac{(n-1) \chi_{0}^{2}}{\chi_{L ; h(\alpha) ; n-1}^{2}}\right)\right\}\right], \quad \chi_{0}^{2} \leq \frac{m^{2}}{n-1} \\
\quad\left[2 \operatorname{Pr}\left(\chi^{2}>\frac{(n-1) \chi_{0}^{2}}{\chi_{L ; h(\alpha) ; n-1}^{2}}\right),\right. \\
\left.\quad \min \left\{1,2 \operatorname{Pr}\left(\chi^{2}>\frac{(n-1) \chi_{0}^{2}}{\chi_{R ; h(\alpha) ; n-1}^{2}}\right)\right\}\right], \quad \chi_{0}^{2}>\frac{m^{2}}{n-1}
\end{array}\right.
$$

So since $\chi^{2}$ follows $\chi_{n-1}^{2}$ distribution with $n-1$ degrees of freedom, (4.7) gives: if $\chi_{0}^{2} \leq \frac{m^{2}}{n-1}$,

$$
\bar{P}[\alpha]=\left[2 F\left(\frac{(n-1) \chi_{0}^{2}}{\chi_{R ; h(\alpha) ; n-1}^{2}}\right), \min \left\{1,2 F\left(\frac{(n-1) \chi_{0}^{2}}{\chi_{L ; h(\alpha) ; n-1}^{2}}\right)\right\}\right],
$$

if $\chi_{0}^{2}>\frac{m^{2}}{n-1}$,

$$
\bar{P}[\alpha]=\left[2\left(1-F\left(\frac{(n-1) \chi_{0}^{2}}{\chi_{L ; h(\alpha) ; n-1}^{2}}\right)\right), \min \left\{1,2\left(1-F\left(\frac{(n-1) \chi_{0}^{2}}{\chi_{R ; h(\alpha) ; n-1}^{2}}\right)\right)\right\}\right]
$$

where $F(x)$ the distribution function of the $\chi_{n-1}^{2}$ distribution. 


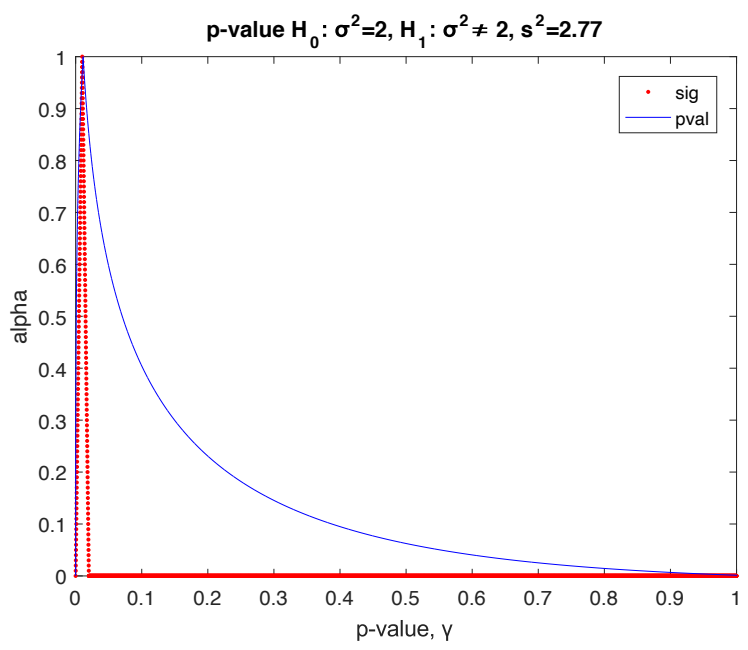

Figure 12. Fuzzy p-value $\bar{P}$ and significance level $\bar{S}$ of the two sided test of Example 6 for the first sample

According to (2.1) the $\alpha$-cuts of the fuzzy $p$-value of the one sided test from the left are

$$
\bar{P}[\alpha]=\left[F\left(\frac{(n-1) \chi_{0}^{2}}{\chi_{R ; h(\alpha) ; n-1}^{2}}\right), F\left(\frac{(n-1) \chi_{0}^{2}}{\chi_{L ; h(\alpha) ; n-1}^{2}}\right)\right]
$$

and according to (2.2), of the one sided test from the right

$$
\bar{P}[\alpha]=\left[1-F\left(\frac{(n-1) \chi_{0}^{2}}{\chi_{L ; h(\alpha) ; n-1}^{2}}\right), 1-F\left(\frac{(n-1) \chi_{0}^{2}}{\chi_{R ; h(\alpha) ; n-1}^{2}}\right)\right]
$$

Using the fuzzy significance level $\bar{S}$ given in (2.4), $H_{0}$ is rejected at significance level $\gamma$ if $\bar{P}<\bar{S}$ and not rejected if $\bar{P}>\bar{S}$ as described is Section 2 .

Example 5. We test the null hypothesis $H_{0}: \sigma^{2}=2$ at significance level $\gamma=0.01$ with alternative $H_{1}: \sigma^{2} \neq 2$ from a random sample of 101 observations with sample variance $s^{2}=1.984$ with two sided fuzzy test which uses:

a) fuzzy $p$-value,

b) fuzzy critical values, developed in [2] and [12].

The crisp value of the test statistic $\chi^{2}$ is found by (4.2) to be

$$
\chi_{0}^{2}=\frac{(101-1) \cdot 1.984}{2}=99.2
$$

So, according to (4.1) the p-value of the crisp test is

$$
\begin{aligned}
p & =\min \left\{2 F_{100}(99.2), 2\left(1-F_{100}(99.2)\right)\right\} \\
& =0.9924>\gamma=0.01
\end{aligned}
$$

Therefore $H_{0}$ is accepted by this test.

a) Implementing (4.8)-(4.9) and fuzzifying the significance level to a triangular 
fuzzy number as in (2.4a) we get the results of Figure 10, from which we see that the core of the fuzzy number $\bar{P}$ is at the right of the core of $\bar{S}$ and their point of intersection has $y_{0}=0.02$, so $\bar{P}>\bar{S}$ with degree of confidence $1-0.02=0.98$. Hence, $H_{0}$ is accepted by this test with degree of acceptance 0.98 .

b) Applying the fuzzy hypothesis test developed in [2] and [12], which uses fuzzy critical values, we get the results of Figure 11, where the core of $\bar{Z}_{0}$ is between the cores of $\overline{C V}_{1}$ and $\overline{C V}_{2}$ and the point of intersection of $\bar{Z}_{0}$ and $\overline{C V}_{1}$ has $y_{1}=0.16$ and of $\bar{Z}_{0}$ and $\overline{C V}_{2}, y_{2}=0.19$. So

$$
\min \left(v\left(\bar{Z}_{0}>\overline{C V}_{1}\right), v\left(\bar{Z}_{0}<\overline{C V}_{2}\right)\right)=\min \left(1-y_{1}, 1-y_{2}\right)=0.81
$$

Therefore, $H_{0}$ is accepted by this test with degree of acceptance $d=0.81$.

Example 6. We test the null hypothesis $H_{0}: \sigma^{2}=2$ at significance level $\gamma=0.01$ with alternative the $H_{1}: \sigma^{2} \neq 2$ (two sided test) using a random sample of 101 observations with sample variance: a) $s^{2}=2.77$, b) $s^{2}=2.83$

For the first sample (variance $s^{2}=2.77$ ) the sample value of the test statistic $\chi^{2}$ is found by (4.2) to be

$$
\chi_{0}^{2}=\frac{(101-1) \cdot 2.77}{2}=138.5,
$$

so according to (4.1), the $p$-value of the crisp test is

$$
\begin{aligned}
p & =\min \left\{2 F_{100}(138.5), 2\left(1-F_{100}(138.5)\right)\right\} \\
& =\min \{1.987,0.013\}=0.013>\gamma=0.01
\end{aligned}
$$

Therefore, $H_{0}$ is accepted by this crisp test.

For the second sample (variance $s^{2}=2.83$ ) (4.2) gives

$$
\chi_{0}^{2}=\frac{(101-1) \cdot 2.83}{2}=141.5,
$$

so according to (4.1), the p-value of the crisp test is

$$
\begin{aligned}
p & =\min \left\{2 F_{100}(141.5), 2\left(1-F_{100}(141.5)\right)\right\} \\
& =\min \{1.992,0.008\}=0.008<\gamma=0.01
\end{aligned}
$$

Therefore, $H_{0}$ is rejected by this crisp test.

a) Implementing (4.8)-(4.9) and fuzzifying the significance level to a triangular fuzzy number as in $(2.4 \mathrm{a})$, we get the results of Figure 12 , where the point of intersection of $\bar{P}$ and $\bar{S}$ has $y_{0}=0.99$, so $v(\bar{P} \approx \bar{S})=0.99$. Hence according to Section 2, we cannot make a decision on rejecting or not $H_{0}$ from this test with any degree of confidence $d \geq 0.01$.

b) For the second sample $(\bar{x}=5.45)$ we plot the fuzzy numbers of $\bar{P}$ and $\bar{S}$ in Figure 13, where their point of intersection has $y_{0}=0.96$, so $v(\bar{P} \approx \bar{S})=0.96$. Therefore, we cannot make a decision on rejecting $H_{0}$ or not from this test with any degree of confidence $d \geq 0.04$. 


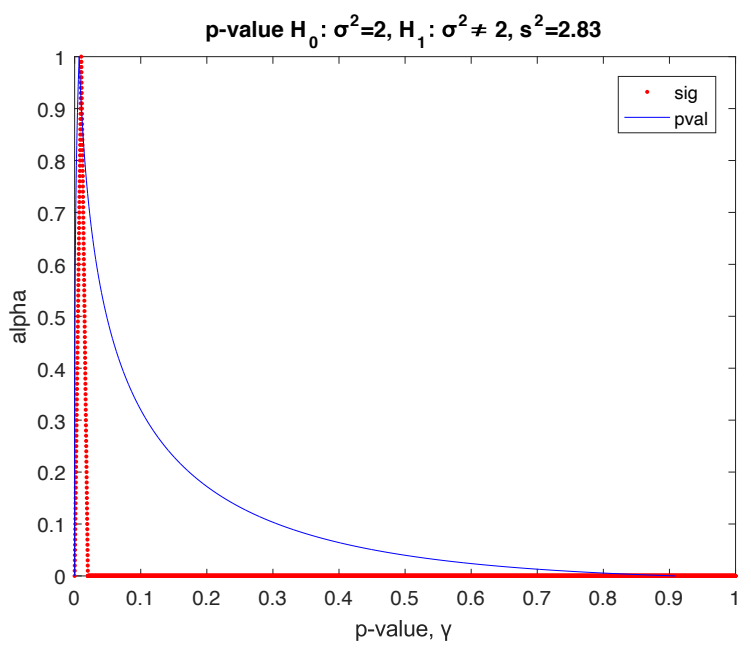

Figure 13. Fuzzy p-value $\bar{P}$ and significance level $\bar{S}$ of the two sided test of Example 6 for the second sample

5. p-value of the Tests of $H_{0}: \sigma_{1}^{2}=\sigma_{2}^{2}$, for the Variances $\sigma_{1}^{2}, \sigma_{2}^{2}$ of two Normal Distributions

We test at significance level $\gamma$ the null hypothesis that the variances $\sigma_{1}^{2}$ and $\sigma_{2}^{2}$ of two normal random variables $X_{1}$ and $X_{2}$ are equal,

$$
H_{0}: \sigma_{1}^{2}=\sigma_{2}^{2}
$$

using two independent random samples of observations of $X_{1}$ and $X_{2}$ of sizes $n_{1}$, $n_{2}$ and variances $s_{1}^{2}, s_{2}^{2}$.

It is known [6] that

$$
\chi_{1}^{2}=\frac{\left(n_{1}-1\right) S_{1}^{2}}{\sigma_{1}^{2}} \sim \chi_{n_{1}-1}^{2} \quad \text { and } \quad \chi_{2}^{2}=\frac{\left(n_{2}-1\right) S_{2}^{2}}{\sigma_{2}^{2}} \sim \chi_{n_{2}-1}^{2}
$$

where $S_{1}^{2}, S_{2}^{2}$ the statistics of the sample variances. So, in the crisp case under the null hypothesis (equal variances) the test statistic

$$
F=\frac{\frac{\chi_{1}^{2}}{n_{1}-1}}{\frac{\chi_{2}^{2}}{n_{2}-1}}=\frac{S_{1}^{2}}{S_{2}^{2}}
$$

follows a $F_{n_{1}-1, n_{2}-1}$ distribution with $n_{1}-1$ degrees of freedom for the numerator and $n_{2}-1$ degrees of freedom for the denominator [6]. Therefore, $H_{0}$ is rejected if $p \leq \gamma$, where according to (1.1) the crisp $p$-value of the test is: for the one sided test from the left,

$$
p=F_{n_{1}-1, n_{2}-1}\left(\frac{s_{1}^{2}}{s_{2}^{2}}\right)
$$


for the one sided test from the right,

$$
p=1-F_{n_{1}-1, n_{2}-1}\left(\frac{s_{1}^{2}}{s_{2}^{2}}\right)
$$

for the two sided test,

$$
p=\min \left\{2 F_{n_{1}-1, n_{2}-1}\left(\frac{s_{1}^{2}}{s_{2}^{2}}\right), 2\left(1-F_{n_{1}-1, n_{2}-1}\left(\frac{s_{1}^{2}}{s_{2}^{2}}\right)\right)\right\}
$$

where $F_{n_{1}-1, n_{2}-1}(x)$ the distribution function of the $F_{n_{1}-1, n_{2}-1}$ distribution. If $p>\gamma$, then $H_{0}$ is not rejected.

In the fuzzy test of $H_{0}$ we use the statistic

$$
\bar{F}=\frac{{\overline{\sigma_{1}^{2}}}_{\overline{\sigma_{2}^{2}}}}{\bar{F} \text { isthe }}
$$

created by the fuzzification of $F$. Since $\bar{F}$ is the ratio of the fuzzy estimators $\bar{\sigma}_{1}^{2}$ and $\bar{\sigma}_{2}^{2}$, its $\alpha$-cuts are found using interval arithmetics for their $\alpha$-cuts, which in our approach are given by (4.4). So, the $\alpha$-cuts of the fuzzy statistic $\bar{F}$ for the given sample are found to be

$$
\begin{aligned}
& \bar{F}[\alpha]=\left[\begin{array}{ll}
\frac{\left(n_{1}-1\right) s_{1}^{2}}{\chi_{R ; h(a) ; n_{1}-1}^{2}}, & \frac{\left(n_{1}-1\right) s_{1}^{2}}{\chi_{L ; h(a) ; n_{1}-1}^{2}} \\
\frac{\left(n_{2}-1\right) s_{2}^{2}}{\chi_{L ; h(a) ; n_{2}-1}^{2}} & \frac{\left(n_{2}-1\right) s_{2}^{2}}{\chi_{R ; h(a) ; n_{2}-1}^{2}}
\end{array}\right] \\
& =\left[\frac{\left(n_{1}-1\right) s_{1}^{2}}{\left(n_{2}-1\right) s_{2}^{2}} \frac{\chi_{L ; h(a) ; n_{2}-1}^{2}}{\chi_{R ; h(a) ; n_{1}-1}^{2}}, \frac{\left(n_{1}-1\right) s_{1}^{2}}{\left(n_{2}-1\right) s_{2}^{2}} \frac{\chi_{R ; h(a) ; n_{2}-1}^{2}}{\chi_{L ; h(a) ; n_{1}-1}^{2}}\right]
\end{aligned}
$$

where $\chi_{L ; h(a) ; n_{1}-1}^{2}$ and $\chi_{R ; h(a) ; n_{2}-1}^{2}$ are defined by (4.5).

The core of $\bar{F}$ is obtained by (5.2) for $\alpha=1$ to be

$$
\frac{\left(n_{1}-1\right) s_{1}^{2}}{\left(n_{2}-1\right) s_{2}^{2}} \frac{\chi_{L ; h(1) ; n_{2}-1}^{2}}{\chi_{R ; h(1) ; n_{1}-1}^{2}}=\frac{\left(n_{1}-1\right) s_{1}^{2} m_{n_{2}-1}}{\left(n_{2}-1\right) s_{2}^{2} m_{n_{1}-1}}
$$

since from $(3.5) h(1)=0.5$, so

$$
\frac{\chi_{L ; h(1) ; n_{2}-1}^{2}}{\chi_{R ; h(1) ; n_{1}-1}^{2}}=\frac{\chi_{L ; 0.5 ; n_{2}-1}^{2}}{\chi_{R ; 0.5 ; n_{1}-1}^{2}}=\frac{F_{n_{2}-1}^{-1}(0.5)}{F_{n_{1}-1}^{-1}(0.5)}=\frac{m_{n_{2}-1}}{m_{n_{1}-1}}
$$

where $m_{n_{1}-1}, m_{n_{2}-1}$ the medians of the $\chi_{n_{1}-1}^{2}$ and $\chi_{n_{2}-1}^{2}$ distributions and $F_{n_{1}-1}^{-1}$, $F_{n_{1}-1}^{-1}$ their inverse distribution function. Hence, if $m=F_{n_{1}-1, n_{2}-1}^{-1}(0.5)$ the median of the $F_{n_{1}-1, n_{2}-1}$ distribution $\left(F_{n_{1}-1, n_{2}-1}^{-1}\right.$ the inverse distribution function of the $F_{n_{1}-1, n_{2}-1}$ distribution)

$$
\frac{\left(n_{1}-1\right) s_{1}^{2} m_{n_{2}-1}}{\left(n_{2}-1\right) s_{2}^{2} m_{n_{1}-1}}<m \Leftrightarrow \frac{s_{1}^{2}}{s_{2}^{2}}<\frac{\left(n_{2}-1\right) m_{n_{1}-1}}{\left(n_{1}-1\right) m_{n_{2}-1}} m
$$

Therefore according to (2.3), the $\alpha$-cuts of the p-value of the two sided test of $H_{0}$ are: 
if $\frac{s_{1}^{2}}{s_{2}^{2}} \leq \frac{\left(n_{2}-1\right) m_{n_{1}-1}}{\left(n_{1}-1\right) m_{n_{2}-1}} m$

$$
\begin{aligned}
\bar{P}[\alpha]= & {\left[2 \operatorname{Pr}\left(F \leq \frac{\left(n_{1}-1\right) s_{1}^{2}}{\left(n_{2}-1\right) s_{2}^{2}} \frac{\chi_{L ; h(a) ; n_{2}-1}^{2}}{\chi_{R ; h(a) ; n_{1}-1}^{2}}\right),\right.} \\
& \left.\min \left\{1,2 \operatorname{Pr}\left(F \leq \frac{\left(n_{1}-1\right) s_{1}^{2}}{\left(n_{2}-1\right) s_{2}^{2}} \frac{\chi_{R ; h(a) ; n_{2}-1}^{2}}{\chi_{L ; h(a) ; n_{1}-1}^{2}}\right)\right\}\right],
\end{aligned}
$$

if $\frac{s_{1}^{2}}{s_{2}^{2}}>\frac{\left(n_{2}-1\right) m_{n_{1}-1}}{\left(n_{1}-1\right) m_{n_{2}-1}} m$,

$$
\begin{aligned}
\bar{P}[\alpha]= & {\left[2 \operatorname{Pr}\left(F \geq \frac{\left(n_{1}-1\right) s_{1}^{2}}{\left(n_{2}-1\right) s_{2}^{2}} \frac{\chi_{R ; h(a) ; n_{2}-1}^{2}}{\chi_{L ; h(a) ; n_{1}-1}^{2}}\right),\right.} \\
& \left.\min \left\{1,2 \operatorname{Pr}\left(F \geq \frac{\left(n_{1}-1\right) s_{1}^{2}}{\left(n_{2}-1\right) s_{2}^{2}} \frac{\chi_{L ; h(a) ; n_{2}-1}^{2}}{\chi_{R ; h(a) ; n_{1}-1}^{2}}\right)\right\}\right]
\end{aligned}
$$

Since $F$ follows the $F_{n_{1}-1, n_{2}-1}$ distribution, (5.3) and (5.4) give:

$$
\text { if } \begin{aligned}
& \frac{s_{1}^{2}}{s_{2}^{2}} \leq \frac{\left(n_{2}-1\right) m_{n_{1}-1}}{\left(n_{1}-1\right) m_{n_{2}-1}} m, \\
& \bar{P}[\alpha]= {\left[2 F_{n_{1}-1, n_{2}-1}\left(\frac{\left(n_{1}-1\right) s_{1}^{2}}{\left(n_{2}-1\right) s_{2}^{2}} \frac{\chi_{L ; h(a) ; n_{2}-1}^{2}}{\chi_{R ; h(a) ; n_{1}-1}^{2}}\right),\right.} \\
&\left.\min \left\{1,2 F_{n_{1}-1, n_{2}-1}\left(\frac{\left(n_{1}-1\right) s_{1}^{2}}{\left(n_{2}-1\right) s_{2}^{2}} \frac{\chi_{R ; h(a) ; n_{2}-1}^{2}}{\chi_{L ; h(a) ; n_{1}-1}^{2}}\right)\right\}\right],
\end{aligned}
$$

if $\frac{s_{1}^{2}}{s_{2}^{2}}>\frac{\left(n_{2}-1\right) m_{n_{1}-1}}{\left(n_{1}-1\right) m_{n_{2}-1}} m$,

$$
\begin{aligned}
\bar{P}[\alpha]= & {\left[2\left(1-F_{n_{1}-1, n_{2}-1}\left(\frac{\left(n_{1}-1\right) s_{1}^{2}}{\left(n_{2}-1\right) s_{2}^{2}} \frac{\chi_{R ; h(a) ; n_{2}-1}^{2}}{\chi_{L ; h(a) ; n_{1}-1}^{2}}\right)\right),\right.} \\
& \left.\min \left\{1,2\left(1-F_{n_{1}-1, n_{2}-1}\left(\frac{\left(n_{1}-1\right) s_{1}^{2}}{\left(n_{2}-1\right) s_{2}^{2}} \frac{\chi_{L ; h(a) ; n_{2}-1}^{2}}{\chi_{R ; h(a) ; n_{1}-1}^{2}}\right)\right)\right\}\right]
\end{aligned}
$$

According to (2.1), the $\alpha$-cuts of the fuzzy $p$-value of the one sided test from the left are

$$
\begin{array}{r}
\bar{P}[\alpha]=\left[F_{n_{1}-1, n_{2}-1}\left(\frac{\left(n_{1}-1\right) s_{1}^{2}}{\left(n_{2}-1\right) s_{2}^{2}} \frac{\chi_{L ; h(a) ; n_{2}-1}^{2}}{\chi_{R ; h(a) ; n_{1}-1}^{2}}\right),\right. \\
\left.F_{n_{1}-1, n_{2}-1}\left(\frac{\left(n_{1}-1\right) s_{1}^{2}}{\left(n_{2}-1\right) s_{2}^{2}} \frac{\chi_{R ; h(a) ; n_{2}-1}^{2}}{\chi_{L ; h(a) ; n_{1}-1}^{2}}\right)\right]
\end{array}
$$

and according to (2.2), of the one sided test from the right

$$
\bar{P}[\alpha]=\left[1-F_{n_{1}-1, n_{2}-1}\left(\frac{\left(n_{1}-1\right) s_{1}^{2}}{\left(n_{2}-1\right) s_{2}^{2}} \frac{\chi_{R ; h(a) ; n_{2}-1}^{2}}{\chi_{L ; h(a) ; n_{1}-1}^{2}}\right),\right.
$$




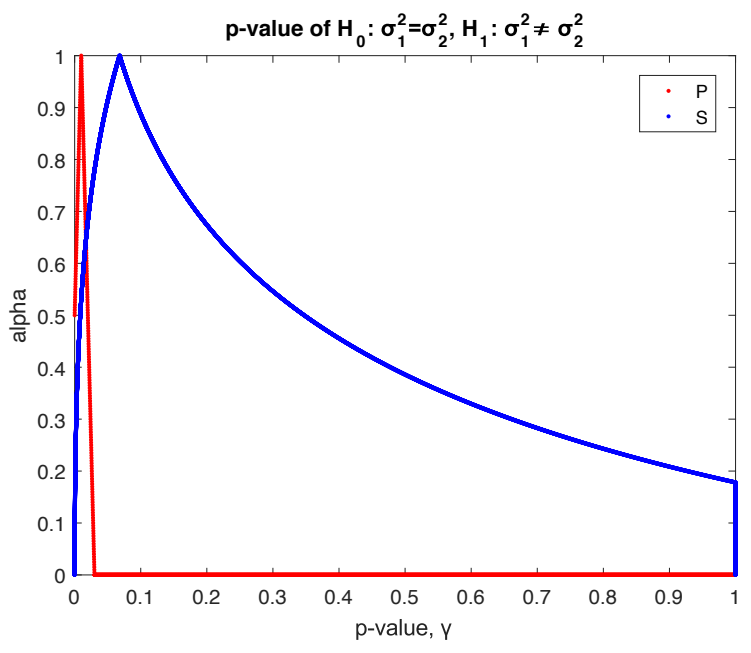

Figure 14. The fuzzy p-value $\bar{P}$ and significance level $\bar{S}$ of the two sided test of Example 7

$$
\left.1-F_{n_{1}-1, n_{2}-1}\left(\frac{\left(n_{1}-1\right) s_{1}^{2}}{\left(n_{2}-1\right) s_{2}^{2}} \frac{\chi_{L ; h(a) ; n_{2}-1}^{2}}{\chi_{R ; h(a) ; n_{1}-1}^{2}}\right)\right]
$$

Example 7. We test at significance level 0.01 the null hypothesis $H_{0}: \sigma_{1}^{2}=\sigma_{2}^{2}$ with alternative $H_{1}: \sigma_{1}^{2} \neq \sigma_{2}^{2}$ (two sided test) using two random samples of $n_{1}=11$ and $n_{2}=13$ observations with sample variances $s_{1}^{2}=0.24$ and $s_{2}^{2}=0.8$ with two sided fuzzy test which uses:

i) fuzzy $p$-value,

ii) fuzzy critical values, developed in [2] and [12].

a) The sample value of the test statistic (5.1) is

$$
F=\frac{s_{1}^{2}}{s_{2}^{2}}=\frac{0.24}{0.8}=0.3
$$

So, the $p$-value of the two sided crisp test is $\left(F_{10,13}(x)\right.$ the distribution function of the $F_{10,13}$ distribution that follows the statistic $F$ ).

$$
\begin{aligned}
p & =\min \left\{2 F_{10,13}(0.3), 2\left(1-F_{10,13}(0.3)\right)\right\} \\
& =\min \{0.064,1.872\}=0.064>\gamma=0.05
\end{aligned}
$$

so $H_{0}$ is not rejected by this test.

Implementing (5.5)-(5.6) and fuzzifying the significance level to a triangular fuzzy number as in (2.4a), we get the results of Figure 14 where the core of $\bar{P}$ is at the right of $\bar{S}$ and their point of intersection has $y_{0}=0.6$. This according to (1.5) means that $v(\bar{P}>\bar{S})=1-0.6=0.4$. So $H_{0}$ is accepted by this test with degree of acceptance $d=0.4$.

b) Applying the fuzzy test developed in [2] and [12], which uses fuzzy critical values, we get the results of Figure 15, where the core of $\bar{Z}_{0}$ is between the cores 


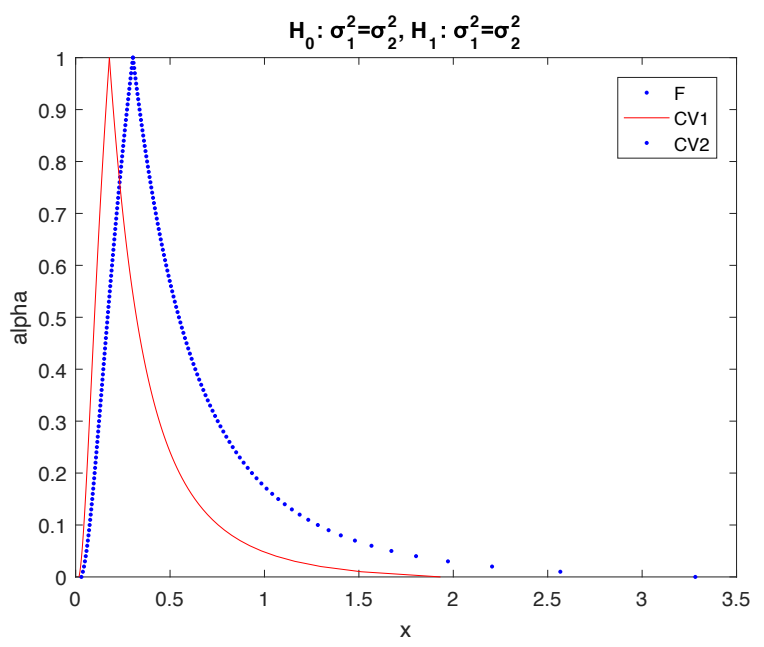

Figure 15 . The fuzzy test statistic $\bar{Z}$ and critical values of the two sided test of Example 7

of $\overline{C V}_{1}$ and $\overline{C V}_{2}$ and the point of intersection of $\bar{Z}_{0}$ and $\overline{C V}_{1}$ has $y_{1}=0.78$ (the point of intersection of $\bar{Z}_{0}$ and $\overline{C V}_{2}$, not shown, has $y_{2}=0.08$ ), so that

$$
\min \left(v\left(\bar{Z}_{0}>\overline{C V}_{1}\right), v\left(\bar{Z}_{0}<\overline{C V}_{2}\right)\right)=\min \left(1-y_{1}, 1-y_{2}\right)=0.22
$$

Therefore as described in Section $2, H_{0}$ is accepted by this test with degree of acceptance $d=0.22$.

\section{Hypotheses Tests for the Mean of a Normal Variable With Unknown Variance}

We test at significance level $\gamma$ the null hypothesis that the mean $\mu$ of a random variable $X$ which follows normal distribution with unknown variance is equal to $\mu_{0}$

$$
H_{0}: \mu=\mu_{0}
$$

using a random sample of $n$ observations of $X$ with sample mean and variance $\bar{x}$ and $s^{2}$.

In the crisp case we test $H_{0}$ using the statistic

$$
T=\frac{\bar{X}-\mu_{0}}{S / \sqrt{n}}
$$

where $\bar{X}$ and $S$ are the statistics of the sample mean and standard deviation. It is known [6] that under the null hypothesis $\left(\mu=\mu_{0}\right) T$ follows $t_{n-1}$ distribution with $n-1$ degrees of freedom. Therefore, $H_{0}$ is rejected if $p \leq \gamma$, where according to (1.1) the crisp $p$-value of the test is: for the one sided test from the left,

$$
p=F_{n-1}\left(t_{0}\right)
$$


for the one sided test from the right,

$$
p=1-F_{n-1}\left(t_{0}\right)
$$

for the two sided test,

$$
p= \begin{cases}2 F_{n-1}\left(t_{0}\right), & t_{0} \leq 0 \\ 2\left(1-F_{n-1}\left(t_{0}\right)\right), & t_{0}>0\end{cases}
$$

where $F_{n-1}(x)$ the distribution function of the $t_{n-1}$ distribution and

$$
t_{0}=\frac{\bar{x}-\mu_{0}}{s / \sqrt{n}}
$$

While if $p>\gamma$, then $H_{0}$ is not rejected.

In the fuzzy case, for the test of $H_{0}$ we use the fuzzy statistic

$$
\bar{T}=\frac{\bar{\mu}-\mu_{0}}{\bar{\sigma} / \sqrt{n}}
$$

which is generated by by substituting $\bar{X}$ and $S$ in (6.1) with the non-asymptotic fuzzy estimators $\bar{\mu}$ and $\bar{\sigma}$ of the mean and standard deviation. As described in [2] the $\alpha$-cuts of $\bar{\mu}$ for the given sample in our approach are [14]

$$
\bar{\mu}[\alpha]=\left[\bar{x}-t_{h(\alpha) ; n-1} \frac{s}{\sqrt{n}}, \bar{x}+t_{h(\alpha) ; n-1} \frac{s}{\sqrt{n}}\right], \quad \alpha \in[0,1]
$$

where $h(a)$ is given by (3.6) and

$$
t_{h(\alpha) ; n-1}=F_{n-1}^{-1}(1-h(\alpha))
$$

According to (4.4) the $\alpha$-cuts of the non-asymptotic fuzzy estimator of the standard deviation for the given sample are

$$
\bar{\sigma}[\alpha]=\left[s \sqrt{\frac{(n-1)}{\chi_{R ; h(\alpha) ; n-1}^{2}}}, s \sqrt{\frac{(n-1)}{\chi_{L ; 1-h(\alpha) ; n-1}^{2}}}\right], \quad \alpha \in[0,1]
$$

where $\chi_{R ; h(\alpha) ; n-1}^{2}$ and $\chi_{L ; h(\alpha) ; n-1}^{2}$ are defined in (4.5).

Using fuzzy number arithmetics from (6.4)-(6.7) follows that the $\alpha$-cuts of the fuzzy statistic $\bar{T}$ for the given sample are

$$
\begin{aligned}
\bar{T}[\alpha] & =\left[\frac{\bar{x}-\mu_{0}-t_{h(\alpha) ; n-1} \frac{s}{\sqrt{n}}}{\frac{1}{\sqrt{n}} s \sqrt{\frac{(n-1)}{\chi_{L ; h(\alpha) ; n-1}^{2}}},} \frac{\bar{x}-\mu_{0}+t_{h(\alpha) ; n-1} \frac{s}{\sqrt{n}}}{\frac{1}{\sqrt{n}} s \sqrt{\frac{(n-1)}{\chi_{R ; h(\alpha) ; n-1}^{2}}}}\right] \\
& =\left[\sqrt{\frac{\chi_{L ; h(\alpha) ; n-1}^{2}}{n-1}\left(t_{0}-t_{h(\alpha)}\right)}, \sqrt{\frac{\chi_{R ; h(\alpha) ; n-1}^{2}}{n-1}\left(t_{0}+t_{h(\alpha)}\right)}\right]
\end{aligned}
$$

where $t_{h(\alpha) ; n-1}$ is given by (6.6).

The median of the $t$ distribution is equal to its mean, which is zero and the core of the $\bar{T}[\alpha]$ is obtained for $\alpha=1$ to be

$$
\sqrt{\frac{\chi_{L ; 0.5 ; n-1}^{2}}{n-1}}\left(t_{0}-t_{h(1) ; n-1}\right)=\sqrt{\frac{m}{n-1}} t_{0}
$$


where $m$ the median of the $\chi_{n-1}^{2}$ distribution, since according to $(3.5) h(1)=0.5$, so $t_{h(1) ; n-1}=t_{0.5 ; n-1}=F_{1-0.5 ; n-1}^{-1}=0$. Therefore, since the statistic $T$ follows $t_{n-1}$ distribution, according to (2.3) the $\alpha$-cuts of the p-value of the two sided test of $H_{0}$ are:

if $\sqrt{\frac{m}{n-1}} t_{0} \leq 0 \Leftrightarrow t_{0} \leq 0$,

$$
\begin{aligned}
\bar{P}[\alpha]= & {\left[2 \operatorname{Pr}\left(T \leq \sqrt{\frac{\chi_{L ; h(\alpha) ; n-1}^{2}}{n-1}}\left(t_{0}-t_{h(\alpha) ; n-1}\right)\right),\right.} \\
& \min \left\{1,2 \operatorname{Pr}\left(T \leq \sqrt{\frac{\chi_{R ; h(\alpha) ; n-1}^{2}}{n-1}}\left(t_{0}+t_{h(\alpha) ; n-1}\right)\right)\right\},
\end{aligned}
$$

if $t_{0}>0$

$$
\begin{aligned}
\bar{P}[\alpha]= & {\left[2 \operatorname{Pr}\left(T \geq \sqrt{\frac{\chi_{R ; h(\alpha) ; n-1}^{2}}{n-1}}\left(t_{0}+t_{h(\alpha) ; n-1}\right)\right),\right.} \\
& \left.\min \left\{1,2 \operatorname{Pr}\left(T \geq \sqrt{\frac{\chi_{L ; h(\alpha) ; n-1}^{2}}{n-1}}\left(t_{0}-t_{h(\alpha) ; n-1}\right)\right)\right\}\right]
\end{aligned}
$$

Since $T$ follows $t_{n-1}$ distribution, (6.8) and (6.9) give:

if $t_{0} \leq 0$,

$$
\begin{aligned}
\bar{P}[\alpha]= & {\left[2 F_{n-1}\left(\sqrt{\frac{\chi_{L ; h(\alpha) ; n-1}^{2}}{n-1}}\left(t_{0}-t_{h(\alpha) ; n-1}\right)\right),\right.} \\
& \left.\min \left\{1,2 F_{n-1}\left(\sqrt{\frac{\chi_{R ; h(\alpha) ; n-1}^{2}}{n-1}}\left(t_{0}+t_{h(\alpha) ; n-1}\right)\right)\right\}\right]
\end{aligned}
$$

if $t_{0}>0$

$$
\begin{aligned}
& \bar{P}[\alpha]=\left[2 \left(1-F_{n-1}\left(\sqrt{\frac{\chi_{R ; h(\alpha) ; n-1}^{2}}{n-1}}\left(t_{0}+t_{h(\alpha) ; n-1}\right)\right),\right.\right. \\
& \left.\quad \min \left\{1,2\left(1-F_{n-1}\left(\sqrt{\frac{\chi_{L ; h(\alpha) ; n-1}^{2}}{n-1}}\left(t_{0}-t_{h(\alpha) ; n-1}\right)\right)\right)\right\}\right]
\end{aligned}
$$

where $F_{n-1}(t)$ the distribution function of the $t_{n-1}$ distribution.

According to (2.1) the $\alpha$-cuts of the fuzzy $p$-value of the one sided test from the left are

$$
\bar{P}[\alpha]=\left[F_{n-1}\left(\sqrt{\frac{\chi_{L ; h(\alpha) ; n-1}^{2}}{n-1}}\left(t_{0}-t_{h(\alpha) ; n-1}\right)\right),\right.
$$




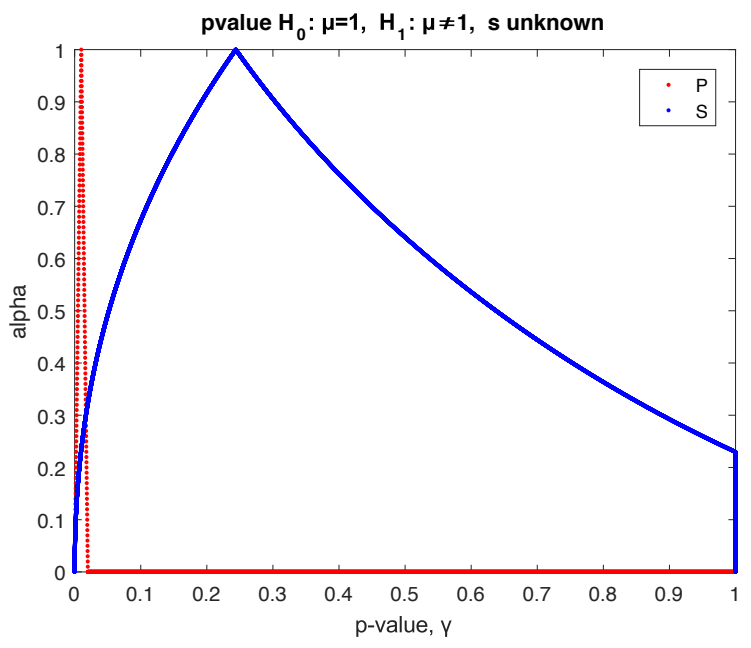

Figure 16. Fuzzy p-value $\bar{P}$ and significance level $\bar{S}$ for the two sided test of $H_{0}: \mu=1$ of Example 8

$$
\left.F_{n-1}\left(\sqrt{\frac{\chi_{R ; h(\alpha) ; n-1}^{2}}{n-1}}\left(t_{0}+t_{h(\alpha) ; n-1}\right)\right)\right]
$$

and according to (2.2) of the one sided test from the right

$$
\begin{aligned}
\bar{P}[\alpha]= & {\left[1-F_{n-1}\left(\sqrt{\frac{\chi_{R ; h(\alpha) ; n-1}^{2}}{n-1}}\left(t_{0}+t_{h(\alpha) ; n-1}\right)\right),\right.} \\
& \left.1-F_{n-1}\left(\sqrt{\frac{\chi_{L ; h(\alpha) ; n-1}^{2}}{n-1}}\left(t_{0}-t_{h(\alpha) ; n-1}\right)\right)\right]
\end{aligned}
$$

Example 8. We test the null hypothesis $H_{0}: \mu=1$ at significance level $\gamma=0.01$ with alternative the $H_{1}: \mu \neq 1$ (two sided test) for the mean $\mu$ of a normal random variable $X$ using a sample of $n=25$ observations with sample mean and variance $\bar{x}=1.32$ and $s^{2}=3.04$.

In the crisp test the sample value of the statistic (6.3) is

$$
t_{0}=\frac{1.32-1}{\sqrt{3.04} / \sqrt{25}}=0.918
$$

so according to (6.2) the $p$-value of the crisp test is

$$
p=2\left(1-F_{25-1}(0.918)\right)=0.3678>\gamma=0.01
$$

Therefore $H_{0}$ is not rejected by the crisp test.

Implementing (6.10)-(6.11) and fuzzifying the significance level to a triangular fuzzy number as in (2.4a), we get Figure 16, where the core of $\bar{P}$ is at the right of 
$\bar{S}$ and the point of intersection of $\bar{P}$ and $\bar{S}$ has $y_{0}=0.31$. So, $H_{0}$ is accepted by this test with degree of acceptance $d=1-0.31=0.69$.

\section{Hypotheses Tests for the Probability of Success $p$ of a Binomial Distribution}

We test at significance level $\gamma$ the null hypothesis that the probability of success $p$ of a binomial distribution is equal to $p_{0}$,

$$
H_{0}: p=p_{0}
$$

using a random sample of observations of size $n$ in which happened $x$ successes.

In the crisp case, we test $H_{0}$ using the statistic [6]

$$
Z=\frac{P-p_{0}}{\sqrt{p_{0} q_{0} / n}}
$$

where $q_{0}=1-p_{0}$ and $P=X / n$ the statistic of the sample proportion of successes. It is known that if $n$ is sufficiently large, then we can use the normal approximation to the binomial, so $Z$ under the null hypothesis follows the standard normal distribution $N(0,1)$. Therefore, $H_{0}$ is rejected if $p$-value $\leq \gamma$, where the crisp $p$-value of the test is found as in Section 3.1 for the sample value of the statistic $Z$,

$$
z_{0}=\frac{\widehat{p}-p_{0}}{\sqrt{p_{0} q_{0} / n}}
$$

and $\widehat{p}=\frac{x}{n}$ is the proportion of successes for the given sample. If $p$-value $>\gamma$, then $H_{0}$ is not rejected.

In the fuzzy case, the test of $H_{0}$ is based on the fuzzy statistic

$$
\bar{Z}=\frac{\bar{p}-p_{0}}{\sqrt{p_{0} q_{0} / n}}
$$

which is generated by substituting $P$ in (7.1) with a fuzzy estimator $\bar{p}$ [2].

In our approach we use the non-asymptotic fuzzy estimator $\bar{p}$, the $\alpha$-cuts of which for the given sample are

$$
\bar{p}[\alpha]=\left[\widehat{p}-z_{h(\alpha)} \sqrt{\frac{\widehat{p} \widehat{q}}{n}}, \widehat{p}+z_{h(\alpha)} \sqrt{\frac{\widehat{p} \hat{q}}{n}}\right], \quad \alpha \in[0,1]
$$

where $\widehat{q}=1-\widehat{p}$ and $h(a)$ and $z_{h(\alpha)}$ are given in (3.5) and (3.6). From (7.1)-(7.4) and interval arithmetics follows that the $\alpha$-cuts of the fuzzy test statistic $\bar{Z}$ for the given sample are

$$
\bar{Z}[\alpha]=\left[z_{0}-z_{h(\alpha)} \sqrt{\frac{\widehat{p} \widehat{q}}{p_{0} q_{0}}}, z_{0}+z_{h(\alpha)} \sqrt{\frac{\widehat{p} \widehat{q}}{p_{0} q_{0}}}\right], \alpha \in[0,1]
$$

Since $Z$ follows the standard normal distribution its median is equal to its mean, which is zero. So according to (2.3), the $\alpha$-cuts of the $p$-value of the two sided 


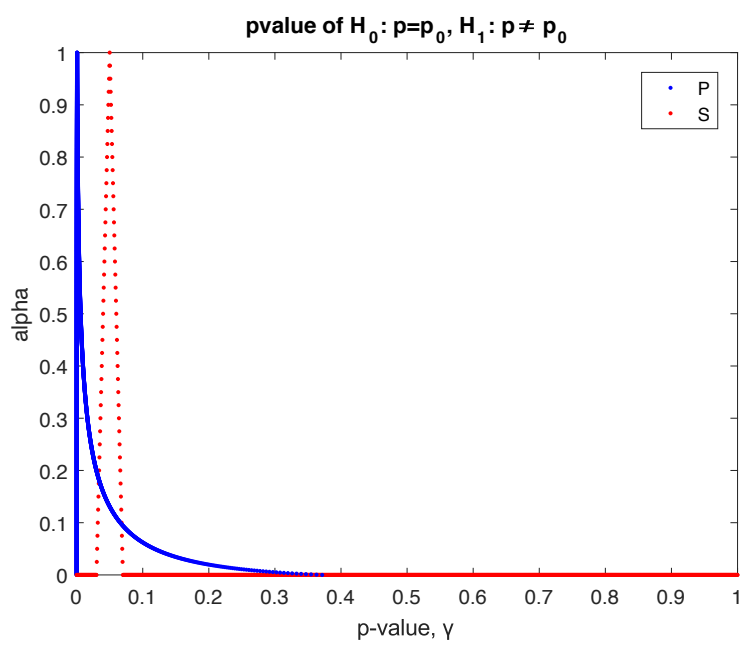

Figure 17 . Fuzzy p-value $\bar{P}$ and significance level $\bar{S}$ for the two sided test of $H_{0}: p=p_{0}$ with $H_{1}: p \neq p_{0}$ of Example 9

test of $H_{0}$ are:

if $z_{0} \leq 0$,

if $z_{0}>0$

$$
\begin{gathered}
\bar{P}[\alpha]=\left[2 \operatorname{Pr}\left(Z \leq z_{0}-z_{h(\alpha)} \sqrt{\frac{\widehat{p} \widehat{q}}{p_{0} q_{0}}}\right)\right. \\
\left.\min \left\{1,2 \operatorname{Pr}\left(Z \leq z_{0}+z_{h(\alpha)} \sqrt{\frac{\widehat{p} \hat{q}}{p_{0} q_{0}}}\right)\right\}\right]
\end{gathered}
$$

$$
\begin{aligned}
\bar{P}[\alpha]= & {\left[2\left(\operatorname{Pr}\left(Z \geq z_{0}+z_{h(\alpha)}\right) \sqrt{\frac{\widehat{p} \widehat{q}}{p_{0} q_{0}}}\right),\right.} \\
& \left.\min \left\{1,2\left(\operatorname{Pr}\left(Z \geq z_{0}-z_{h(\alpha)} \sqrt{\frac{\widehat{p} \widehat{q}}{p_{0} q_{0}}}\right)\right)\right\}\right]
\end{aligned}
$$

Since $Z$ follows the standard normal distribution, (7.6) and (7.7) give: if $z_{0} \leq 0$

$$
\begin{gathered}
\bar{P}[\alpha]=\left[2 \Phi\left(z_{0}-z_{h(\alpha)} \sqrt{\frac{\widehat{p} \widehat{q}}{p_{0} q_{0}}}\right),\right. \\
\min \left\{1,2 \Phi\left(z_{0}+z_{h(\alpha)} \sqrt{\frac{\widehat{p} \widehat{q}}{p_{0} q_{0}}}\right)\right\},
\end{gathered}
$$

if $z_{0}>0$

$$
\bar{P}[\alpha]=\left[2\left(1-\Phi\left(z_{0}+z_{h(\alpha)} \sqrt{\frac{\widehat{p} \widehat{q}}{p_{0} q_{0}}}\right)\right),\right.
$$




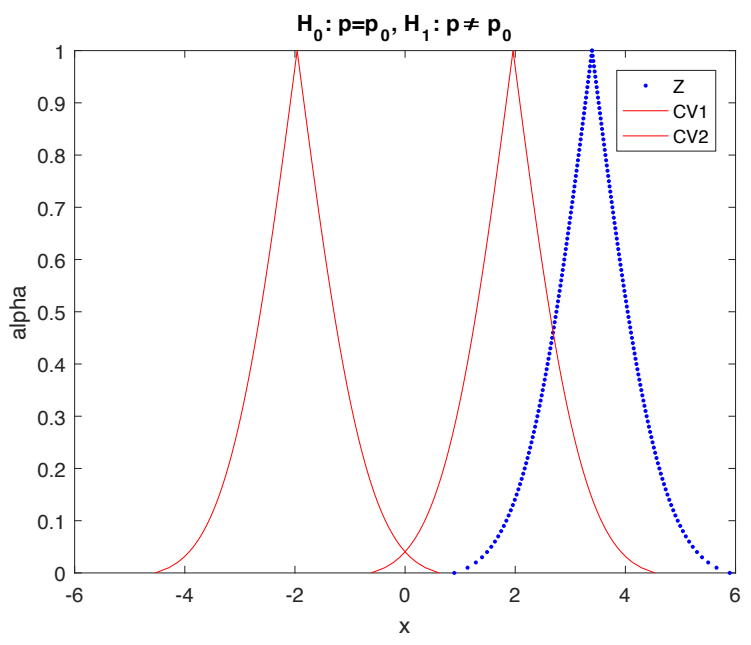

Figure 18. The fuzzy test statistic $Z$ and critical values for the two sided test of Example 9

$$
\left.\min \left\{1,2\left(1-\Phi\left(z_{0}-z_{h(\alpha)} \sqrt{\frac{\widehat{p} \widehat{q}}{p_{0} q_{0}}}\right)\right)\right\}\right]
$$

where $\Phi(z)$ the distribution function of the standard normal distribution and $z_{0}$ and $z_{h(\alpha)}$ are given by (7.2) and (3.6).

According to (2.1), the $\alpha$-cuts of the fuzzy $p$-value of the one sided test from the left are

$$
\bar{P}[\alpha]=\left[\Phi\left(z_{0}-z_{h(\alpha)} \sqrt{\frac{\widehat{p} \widehat{q}}{p_{0} q_{0}}}\right), \Phi\left(z_{0}+z_{h(\alpha)} \sqrt{\frac{\widehat{p} \widehat{q}}{p_{0} q_{0}}}\right)\right]
$$

and according to (2.2) of the one sided test from the right,

$$
\bar{P}[\alpha]=\left[1-\Phi\left(z_{0}+z_{h(\alpha)} \sqrt{\frac{\widehat{p} \widehat{q}}{p_{0} q_{0}}}\right), 1-\Phi\left(z_{0}-z_{h(\alpha)} \sqrt{\frac{\widehat{p} \widehat{q}}{p_{0} q_{0}}}\right)\right]
$$

Example 9. a) We test at significance level $\gamma=0.05$ the null hypothesis that the probability of success $p$ of a binomial distribution is equal to $p_{0}=0.5$

$$
H_{0}: p=0.5
$$

with alternative $H_{1}: p \neq 0.5$ (two sided test) using a random sample of $n=200$ observations in which happened 124 successes with two sided fuzzy test which uses: i) fuzzy $p$-value,

ii) fuzzy critical values, developed in [2] and [12].

b) We test $H_{0}$ at significance level $\gamma=0.05$ with alternative $H_{1}: p>0.5$ using the same sample. 
a) In the crisp case we test $H_{0}$ evaluating the sample value (7.2) of the statistic $Z$ for $\widehat{p}=\frac{124}{200}=0.62$

$$
z_{0}=\frac{0.62-0.5}{\sqrt{0.62(1-0.62) / 200}}=3.5
$$

So, the crisp $p$-value of the test is found as in Section 3.1 to be

$$
p \text {-value }=2(1-\Phi(3.5))=0.0005<\gamma=0.05,
$$

Therefore $H_{0}$ is rejected by the crisp test.

i) Implementing (7.8)-(7.9) and fuzzifying the significance level to a triangular fuzzy number as in (3.11), we get the result of Figure 17, where the core of $\bar{P}$ is at the left of the core $\bar{S}$ and their point of intersection has $y_{0}=0.18$. So according to Section $2, H_{0}$ is rejected by this test with degree of rejection $d=1-0.18=0.82$. ii) Applying the fuzzy test developed in [2] and [12], which uses fuzzy critical values, we get the results of Figure 18, where the core of $\bar{Z}_{0}$ is at the right of the core of $\overline{C V}_{2}$ and their point of intersection has $y_{1}=0.45$. So according to Section $2, H_{0}$ is rejected by this test with degree of rejection $d=1-0.45=0.55$.

b) For the one sided test from the right, as in Section 3.1 the crisp $p$-value of the test is

$$
p \text {-value }=1-\Phi(3.5)=0.00025<\gamma=0.05,
$$

so $H_{0}$ is rejected by the crisp test.

Implementing (7.11) and (3.11) for $z_{0}$ of (7.2) we get the results of Figure 19, where the core of the fuzzy number $\bar{P}$ is at the left of the core of $\bar{S}$ and the point of intersection of $\bar{P}$ and $\bar{S}$ has $y_{0}=0.11$, so the truth value of $\bar{P}<\bar{S}$ is $1-0.11=0.89$. Therefore, $H_{0}$ is rejected by the one sided test with degree of rejection 0.89 .

\section{Hypotheses Tests of $H_{0}: p_{1}=p_{2}$ for the Probabilities of Success $p_{1}, p_{2}$ of Two Binomial Distributions}

We test at significance level $\gamma$ the null hypothesis that the probabilities of success $p_{1}$ and $p_{2}$ of two binomial distributions are equal,

$$
H_{0}: p_{1}=p_{2}
$$

using two random samples of observations of sizes $n_{1}$ and $n_{2}$ in which happened $x_{1}$ and $x_{2}$ successes respectively .

In the crisp case we test $H_{0}$ using the statistic [6]

$$
Z=\frac{P_{1}-P_{2}}{s_{p}}
$$

where $P_{1}=\frac{X_{1}}{n_{1}}, P_{2}=\frac{X_{2}}{n_{2}}$ the statistics of the sample proportions of successes,

$$
s_{p}=\sqrt{\widehat{p} \widehat{q}\left(\frac{1}{n_{1}}+\frac{1}{n_{2}}\right)}
$$




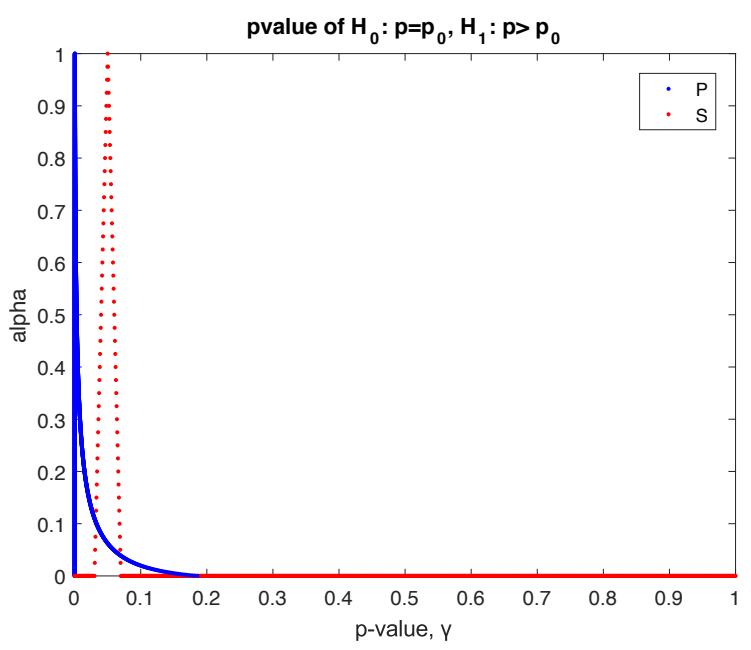

Figure 19. Fuzzy p-value $\bar{P}$ and significance level $\bar{S}$ for the one sided test of $H_{0}: p=p_{0}$ with $H_{1}: p>p_{0}$ of Example 9

and

$$
\widehat{p}=\frac{x_{1}+x_{2}}{n_{1}+n_{2}}
$$

a "poolled" estimator of $p$ under $H_{0}$ and $\widehat{q}=1-\widehat{p}$.

It is known that if $n_{1}$ and $n_{2}$ are sufficiently large, then we can use the normal approximation to the binomial [6]. Then under the null hypothesis $\left(p_{1}=p_{2}\right), Z$ follows the standard normal distribution $N(0,1)$, so $H_{0}$ is rejected if $p$-value $\leq \gamma$, where the crisp $p$-value of the test is found as in Section 3.1 for the sample value of the test statistic (8.1)

$$
z_{0}=\frac{\widehat{p}_{1}-\widehat{p}_{2}}{s_{p}}
$$

where $\widehat{p}_{1}=\frac{x_{1}}{n_{1}}, \widehat{p}_{2}=\frac{x_{2}}{n_{2}}$ the proportions of successes in the given samples.

In the fuzzy case the test of $H_{0}$ is based on the fuzzy statistic

$$
\bar{Z}=\frac{\bar{p}_{12}}{s_{p}}
$$

which is generated by substituting the difference $P_{1}-P_{2}$ in (8.1) with a fuzzy estimator $\bar{p}_{12}[2]$.

In our approach we use the non-asymptotic fuzzy estimator $\bar{p}_{12}$ which is constructed in [2] and generalized in [12], the $\alpha$-cuts of which for the given samples are

$$
\bar{p}_{12}[\alpha]=\left[\widehat{p}_{1}-\widehat{p}_{2}-z_{h(\alpha)} s_{0}, \widehat{p}_{1}-\widehat{p}_{2}+z_{h(\alpha)} s_{0}\right]
$$


where $\widehat{q}_{1}=1-\widehat{p}_{1}, \widehat{q}_{2}=1-\widehat{p}_{2}$ and

$$
s_{0}=\sqrt{\frac{\widehat{p}_{1} \widehat{q}_{1}}{n_{1}}+\frac{\widehat{p}_{2} \widehat{q}_{2}}{n_{2}}}
$$

From (8.1)-(8.7) and interval arithmetics follows that the $\alpha$-cuts of the fuzzy statistic $\bar{Z}$ for the given samples are

$$
\bar{Z}_{0}[\alpha]=\left[z_{0}-z_{h(\alpha)} \frac{s_{0}}{s_{p}}, z_{0}+z_{h(\alpha)} \frac{s_{0}}{s_{p}}\right], \quad \alpha \in[0,1]
$$

Since $Z$ follows the standard normal distribution, its median is equal to its mean, which is zero. So according to (2.3), the $\alpha$-cuts of the fuzzy $p$-value of two sided test are:

if $z_{0} \leq 0$,

$$
\bar{P}[\alpha]=\left[2 \Phi\left(z_{0}-z_{h(\alpha)} \frac{s_{0}}{s_{p}}\right), \min \left\{1,2 \Phi\left(z_{0}+z_{h(\alpha)} \frac{s_{0}}{s_{p}}\right)\right\}\right],
$$

if $z_{0}>0$,

$$
\begin{aligned}
\bar{P}[\alpha] & =\left[2\left(1-\Phi\left(z_{0}+z_{h(\alpha)} \frac{s_{0}}{s_{p}}\right)\right),\right. \\
& \left.\min \left\{1,2\left(1-\Phi\left(z_{0}-z_{h(\alpha)} \frac{s_{0}}{s_{p}}\right)\right)\right\}\right]
\end{aligned}
$$

where $\Phi(z)$ the distribution function of the standard normal distribution and $z_{0}, s_{p}, s_{0}$ and $z_{h(\alpha)}$ are given by (8.4), (8.2), (8.7) and (3.6).

According to (2.1) the $\alpha$-cuts of the fuzzy $p$-value of the one sided test from the left are

$$
\bar{P}[\alpha]=\left[\Phi\left(z_{0}-z_{h(\alpha)} \frac{s_{0}}{s_{p}}\right), \Phi\left(z_{0}+z_{h(\alpha)} \frac{s_{0}}{s_{p}}\right)\right]
$$

and according to (2.2) of the one sided test from the right,

$$
\bar{P}[\alpha]=\left[\left(1-\Phi\left(z_{0}+z_{h(\alpha)} \frac{s_{0}}{s_{p}}\right)\right), 1-\left(1-\Phi\left(z_{0}-z_{h(\alpha)} \frac{s_{0}}{s_{p}}\right)\right)\right]
$$

Example 10. We test at significance level $\gamma=0.05$ the null hypothesis that the probabilities of success $p_{1}$ and $p_{2}$ of two binomial distributions are equal $H_{0}$ : $p_{1}=p_{2}$ with alternative $H_{1}: p_{1} \neq p_{2}$ (two sided test) using two random samples of observations of sizes $n_{1}=250$ and $n_{2}=400$ in which occurred $x_{1}=40$ and $x_{2}=55$ successes respectively.

In the crisp case we test $H_{0}$ evaluating the value of the statistic (8.1) for the given sample, where

$$
\widehat{p}_{1}=\frac{40}{250}=0,16, \quad \widehat{p}_{2}=\frac{55}{400}=0.138
$$

and

$$
\widehat{p}=\frac{40+55}{250+400}=0.146
$$




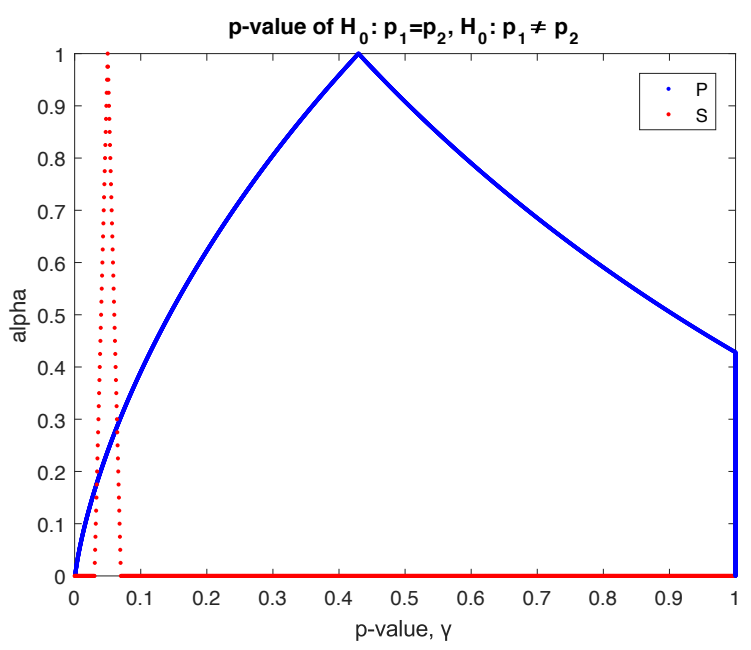

Figure 20. Fuzzy p-value $\bar{P}$ and significance level $\bar{S}$ for the two sided test of $H_{0}: p_{1}=p_{2}$ of Example 10

So from (8.2) we get

$$
s_{p}=\sqrt{0.146(1-0.146)\left(\frac{1}{250}+\frac{1}{400}\right)}=0.029
$$

and from (8.4)

$$
z_{0}=\frac{0.16-0.138}{0.029}=0.759
$$

Thus, the crisp $p$-value of the test is

$$
p \text {-value }=2(1-\Phi(0.759))=0.448>\gamma=0.05
$$

so $H_{0}$ is not rejected by the crisp test.

Implementing (8.9)-(8.10) and fuzzifying the significance level to a triangular fuzzy number as in (3.11), we get the result of Figure 20, where the core of $\bar{P}$ is at the right of the core $\bar{S}$ and their point of intersection has $y_{0}=0.28$. So according to Section 2, $H_{0}$ is accepted by this test with degree of acceptance $d=1-0.28=0.72$.

\section{Conclusions}

If the value of the test statistic of a hypothesis test is close to the critical values of the test, then the crisp test is unstable, since a very small change in the sample may lead from rejection to no rejection of $H_{0}$ or vice-versa, as in Examples 3 and 6. Whereas, our approach, which uses non-asymptotic fuzzy estimators for the construction of the fuzzy $p$-value and a degree of rejection or acceptance of the null hypothesis $H_{0}$, gives better results than the crisp test, since it gives us the possibility to make a decision on a partial rejection or acceptance of $H_{0}$, as shown 
in Examples 3 and 6 where we get a very low degree of rejection or acceptance of the null hypothesis, which means "no decision".

The advantage of our approach compared to the approach of [13] is that it tests crisp hypotheses using fuzziness, which leads to a degree of rejection or acceptance of the null hypothesis, whereas the tests of [13] give crisp results when applied to crisp hypotheses, as explained in its examples.

In the crisp statistics the hypotheses tests which use critical values are equivalent to the tests which use $p$-value. This does not happen in the fuzzy hypotheses tests, since in general the tests which use fuzzy critical values give different degrees of rejection or acceptance of $H_{0}$ than the tests which use fuzzy $p$-value. The two tests give similar results only in cases in which the $p$-value of the test is close to the significance level (or equivalently if the value of the test statistic is close to a critical value), but in the other cases the former gives significantly lower degree of rejection or acceptance than the latter (Examples 2,4,5,7 and 9). Characteristic is the case of the hypotheses tests of the Examples 2 and 5, in which the crisp $p$-value is one (the value of the test statistic is in the center of the acceptance region), which is the best case of acceptance. As shown in these examples, the tests that use fuzzy critical values give significantly lower degree of acceptance of $H_{0}$ than the expected, which is a value close to one, $(d=0.68$ in Example 2 and $d=0.81$ in Example 5), whereas the tests that use fuzzy $p$-value give degree of acceptance of $H_{0}$ near to one $(d=0.95$ in Example 2 and $d=0.98$ in Example $5)$. So, since the tests which use fuzzy $p$-value are in much better agreement with the crisp hypotheses tests than the tests which use fuzzy critical values in [2] and [12], we can conclude that the former give more reliable results than the latter.

\section{References}

1. Buckley, J. J.: Uncertain probabilities II: the continuous case, Soft. Comput. 8 (2004) 193199.

2. Buckley, J. J.: Fuzzy statistics, Springer-Verlag, Berlin, 2004.

3. Dubois, D. and Prade, H.: Ranking of fuzzy numbers in the setting of possibility theory, Information Science 30 (1983) 183-224.

4. Dubois, D. and Prade, H.: Fuzzy Sets and Systems: Theory and applications, Academic Press, 1980.

5. Filzmoser, P. and Viertl, R.: Testing hypotheses with fuzzy data: The fuzzy p-value, Metrika 59 (2004) 21-29.

6. Hogg, R. V. and Tanis, E. A.: Probability and Statistical Inference, 6th edn, Prentice Hall, Upper Saddle River, N.J., 2001.

7. Holena, M.: Fuzzy hypotheses testing in a framework of fuzzy logic, Fuzzy Sets and Systems 145 (2004) 229-252.

8. Klir, G. and Yuan, B.: Fuzzy Sets and Fuzzy Logic: Theory and Applications. Prentice Hall, Upper Saddle River, NJ., 1995.

9. Lee, H. and Lee, J. H.: A method for ranking fuzzy numbers and its application to a decisionmaking, IEEE Transactions on Fuzzy Systems 7 (6) (1999) 677-685.

10. Lee, S., Lee, H., and Lee, D.: Ranking the sequences of fuzzy values, Information Sciences 160 (2004) 41-52.

11. Mylonas, N. and Papadopoulos, B.: Hypotheses tests using non-asymptotic fuzzy estimators and fuzzy critical values, Proceedings of the 16th International Conference on Artificial Intelligence Applications and Innovations, 584, (2020) 157-166.

12. Mylonas, N. and Papadopoulos, B.: Fuzzy hypotheses tests for crisp data using nonasymptotic fuzzy estimators and a degree of rejection or acceptance (under review). 
13. Parchami, A. and Taheri, S, Mahmoud: Fuzzy p-value in testing fuzzy hypotheses with crisp data, Stat. papers 51 (2010) 209-226.

14. Sfiris, D. and Papadopoulos, B.: Non-asymptotic fuzzy estimators based on confidence intervals, Information Sciences 279 (2014) 446-459.

15. Zadeh, L. A.: Fuzzy sets, Information and Control 8 (3) (1965) 338-353.

Nikos Mylonas: Section of Mathematics and Informatics, Department of Civil Engineering, School of Engineering, Democritus University of Thrace, Kimeria, Xanthi, 67100, GReECE

E-mail address: nimylona@civil.duth.gr

Basil K. Papadopoulos: Section of Mathematics and Informatics, Department of Civil Engineering, School of Engineering, Democritus University of Thrace, Kimeria, XANthi, 67100, Greece

E-mail address: papadob@civil.duth.gr 